St. Norbert College

Digital Commons @ St. Norbert College

Faculty Creative and Scholarly Works

$1-19-2012$

Forest elephant mitochondrial genomes reveal that elephantid diversification in Africa tracked climate transitions

Adam L. Brandt

Yasuko Ishida

Nicholas Georgiadis

Alfred L. Roca

Follow this and additional works at: https://digitalcommons.snc.edu/faculty_staff_works 


\title{
Forest elephant mitochondrial genomes reveal that elephantid diversification in Africa tracked climate transitions
}

\author{
Type of submission: Original Article
}

Adam L. Brandt ${ }^{1}$, Yasuko Ishida ${ }^{1}$, Nicholas J. Georgiadis ${ }^{2}$ and Alfred L. Roca ${ }^{1,3^{*}}$

${ }^{1}$ Department of Animal Sciences, University of Illinois at Urbana-Champaign, Urbana, IL 61801 USA

${ }^{2}$ Director, Bole and Klingenstein Foundation, Cody, WY 82414 USA

${ }^{3}$ Institute for Genomic Biology, University of Illinois at Urbana-Champaign, Urbana, IL 61801

USA

*Corresponding Author:

Alfred L. Roca

432 Animal Sciences Laboratory

Department of Animal Sciences

University of Illinois at Urbana-Champaign

Urbana, Illinois 61801

Office phone: $217-244-8853$

Department fax: 217-333-8286

Email: roca@illinois.edu

Keywords: Asian elephant, coalescent, molecular clock, Proboscidea, savanna elephant, woolly mammoth

Running title: Forest elephant mitogenomes 


\section{Abstract}

Among elephants, the phylogeographic patterns of mitochondrial (mt) and nuclear markers are

49 often incongruent. One hypothesis attributes this to sex differences in dispersal and in the

50 variance of reproductive success. We tested this hypothesis by examining the coalescent dates of

51 genetic markers within elephantid lineages, predicting that lower dispersal and lower variance in

52 reproductive success among females would have increased mtDNA relative to nuclear coalescent

53 dates. We sequenced the mitochondrial genomes of two forest elephants, aligning them to

54 mitogenomes of African savanna and Asian elephants, and of woolly mammoths, including the

55 most divergent mitogenomes within each lineage. Using fossil calibrations, the divergence

56 between African elephant $\mathrm{F}$ and $\mathrm{S}$ clade mitochondrial genomes (originating in forest and

57 savanna elephant lineages, respectively) was estimated as 5.5 million years ago (Mya). We

58 estimated that the (African) ancestor of the mammoth and Asian elephant lineages diverged 6.0

59 Mya, indicating that four elephantid lineages had differentiated in Africa by the Miocene-

60 Pliocene transition, concurrent with drier climates. The coalescent date for forest elephant

61 mtDNAs was ca. 2.4 Mya, suggesting that the decrease in tropical forest cover during the

62 Pleistocene isolated distinct African forest elephant lineages. For all elephantid lineages, the ratio

63 of mtDNA to nuclear coalescent dates was much greater than 0.25 . This is consistent with the

64 expectation that sex differences in dispersal and in variance of reproductive success would have

65 increased the effective population size of mtDNA relative to nuclear markers in elephantids,

66 contributing to the persistence of incongruent mtDNA phylogeographic patterns. 
Introduction

A number of studies have indicated that the African savanna or bush elephant (Loxodonta africana) is a distinct species from the African forest elephant (L. cyclotis) (Groves \& Grubb

71 2000; Grubb et al. 2000; Roca et al. 2001; Comstock et al. 2002; Roca et al. 2005; Rohland et al.

72 2010). Analyses of DNA sequences have demonstrated that little or no nuclear gene flow occurs

73 between forest elephant and savanna elephant populations, providing evidence that the two

74 groups would satisfy the definition, under the biological species concept, that "species are groups

75 of interbreeding natural populations that are reproductively isolated from other such groups"

76 (Mayr 1963; Meier \& Wheeler 2000; Roca et al. 2001; Roca et al. 2005; Roca et al. 2007; Petit

77 \& Excoffier 2009). Corroborating this evidence are reports that forest and savanna elephants can

78 be completely distinguished morphologically using a discriminant function (Groves \& Grubb

79 2000), that between forest and savanna elephants Fst values greater than 0.90 have been

80 estimated for nuclear sequences and microsatellites (Roca et al. 2001; Comstock et al. 2002),

81 that phylogenies inferred using nuclear markers place forest and savanna elephants into

82 reciprocally monophyletic clades (Roca et al. 2001; Comstock et al. 2002), and that the

83 divergence between them has been estimated as 2.6 to 5.6 Mya using nuclear markers (Rohland

84 et al. 2010).

85 Mitochondrial (mt) DNA phylogeographic patterns among elephantids are often

86 discordant with those of nuclear DNA markers or morphology (Debruyne 2005; Roca et al.

87 2005; Enk et al. 2011; Ishida et al. 2011; Lei et al. 2011). Among African elephants, mtDNA

88 forms two deeply distinctive clades, designated clades F and S (Debruyne 2005), originating in

89 forest and savanna elephants, respectively (Ishida et al. 2011). Forest elephants do not carry S 
90 clade mtDNA, while some nuclear alleles that are common or fixed across savanna elephants are

91 not found in forest elephants (Roca et al. 2005; Ishida et al. 2011), suggesting that forest

92 elephant populations may be completely or almost completely isolated from gene flow from

93 savanna elephant populations. By contrast, many African savanna elephant individuals and

94 populations are known to carry F clade mtDNA (Debruyne 2005; Roca et al. 2005; Lei et al.

95 2008), although almost all of these show no evidence of carrying forest elephant nuclear alleles

96 (Roca et al. 2005; Lei et al. 2009). This mito-nuclear incongruence has suggested that species

97 isolation mechanisms exist that largely prevent hybrid males, but not hybrid females, from

98 reproducing successfully among savanna elephants (Roca et al. 2005; Roca \& O'Brien 2005;

99 Roca et al. 2007). Nonetheless, based on both simulations and empirical surveys of interspecies

100 gene flow patterns (Currat et al. 2008; Petit \& Excoffier 2009), male-mediated dispersal in

101 savanna elephants (Archie et al. 2008) would be expected to facilitate the widespread

102 introgression of mtDNA but not nuclear alleles from forest to savanna elephant populations.

103 Among Asian elephants (Elephas maximus), two deeply divergent mtDNA clades

104 separated 1.6 to 2.1 Mya. These mtDNA clades do not appear to correspond to recognized

105 subspecies subdivisions (Deraniyagala 1955), or to nuclear genetic subdivisions in Asian

106 elephants (Lei et al. 2011), but may instead reflect the impact of Pleistocene glacial cycles on the

107 climate and habitats of Asia (Fernando et al. 2000; Fleischer et al. 2001; Fernando et al. 2003;

108 Vidya et al. 2009). In an extinct elephantid, the woolly mammoth (Mammuthus primigenius),

109 two clades with ca. 1 to 2 Mya divergence have been detected (Gilbert et al. 2008). One clade

110 appears to be more geographically restricted than the other, and to have gone extinct earlier

111 (Gilbert et al. 2008). The two woolly mammoth mtDNA clades, and further subdivisions

112 detected within them, have provided the framework for hypotheses involving the potential 
113 effects of speciation events, migrations, habitat changes, and glacial cycles on woolly mammoths

114 (Barnes et al. 2007; Debruyne et al. 2008; Gilbert et al. 2008; Miller et al. 2008). Recently,

115 woolly mammoth mtDNA was found to have introgressed into a morphologically distinctive

116 species, the Columbian mammoth (Mammuthus columbi) (Enk et al. 2011).

117 Sex differences in dispersal and in the variance of reproductive success have been well

118 documented in living elephantids (Archie et al. 2007; Hollister-Smith et al. 2007; Roca et al.

119 2007; Poole et al. 2011). Elephant females reaching maturity remain with their natal core social

120 group or "herd" (Archie et al. 2007; Hollister-Smith et al. 2007) and females do not typically

121 migrate between herds (Archie et al. 2007; Hollister-Smith et al. 2007), thus the mitochondrial

122 genome is necessarily coupled to the geographic range of the core social group. By contrast,

123 males leave the natal herd and mediate gene flow between herds and across the landscape

124 (Archie et al. 2007; Hollister-Smith et al. 2007; Roca et al. 2007; Poole et al. 2011).

125 Furthermore, male-male competition is intense, and in any generation only a small proportion of

126 males are reproductively successful (Archie et al. 2007; Hollister-Smith et al. 2007; Rasmussen

127 et al. 2008). We have previously formulated the hypothesis that these well-documented sex

128 differences in elephants have enabled the observed phylogeographic incongruence in mito-

129 nuclear patterns (Ishida et al. 2011).

130 Since lower female dispersal and higher variance in male reproductive success would

131 have increased mtDNA effective population sizes relative to those of nuclear loci (Hoelzer 1997;

132 Hedrick 2007), then if the matrilocal and matrilineal social structure and intense male

133 reproductive competition are responsible for the observed mito-nuclear incongruence (Ishida et

134 al. 2011), one would predict that the ratio of coalescent dates for mtDNA relative to those of

135 nuclear loci would have a value greater than the 0.25 expected in populations without sex 
136 differences in dispersal or in variance in reproductive success. Here, we examine the coalescent

137 dates of mtDNA and nuclear loci in elephantids to test our expectation that sex differences in

138 dispersal and in the variance of reproductive success have led to disproportionately high mtDNA

139 effective population sizes and coalescent dates relative to those of nuclear markers. We

140 generated full mitochondrial genome sequences for Loxodonta cyclotis, sequencing two forest

141 elephants that carried haplotypes corresponding to the two most divergent mtDNA subclades

142 within the species (Debruyne 2005; Roca et al. 2005). We aligned them to mitogenomes

143 representing the most divergent mtDNA clades within each of the other elephantid lineages. We

144 examined the relationships of forest elephants to other elephantid taxa, and estimated divergence

145 and coalescent dates using fossil calibrations. We contrasted the ratio of mtDNA to nuclear

146 coalescent dates within and across elephantid taxa. Finally, we compared our date estimates to

147 the timing of climate transitions, finding that elephantid evolution in Africa may have been

148 driven by drier climates at the end of the Miocene and by the initiation of glacial cycles at the 149 start of the Pleistocene.

151 Materials and Methods

Elephant samples and DNA extraction. The study was conducted in compliance with the

154 University of Illinois Institutional Animal Care and Use Committed (IACUC) approved protocol

155 number 09036. Samples were obtained in full compliance with required CITES (Convention on

156 International Trade in Endangered Species of Wild Fauna and Flora) and other permits. Two

157 forest elephant DNA samples were used. One was extracted from tissue collected using a biopsy

158 dart from a female forest elephant (designated Lcy-DS1534 or DS1534) in the Dzanga Sangha 
159 Forest Reserve of the Central African Republic (Georgiadis et al. 1994). The other was extracted

160 from a blood sample of "Coco," a male forest elephant (Loxodonta cyclotis) from Sierra Leone,

161 designated Lcy-SL0001 or SL0001, generously provided by the Paris Zoo (Parc Zoologique de

162 Paris-Vincennes, France). Nuclear DNA sequences had previously established that both

163 individuals were forest elephants (Roca et al. 2001; Roca et al. 2005; Capelli et al. 2006; Ishida

164 et al. 2011); mtDNA sequences had established that SL0001 was from a sub-clade of mtDNA

165 found in West Africa (Barriel et al. 1999; Eggert et al. 2002; Debruyne et al. 2003; Debruyne

166 2005). DNA was extracted using a kit from Qiagen (SL0001), or a standard phenol-chloroform

167 method (DS1534) (Sambrook et al. 1989).

168 Oligonucleotide primers. The mitochondrial genome was amplified in eight long

169 overlapping fragments. We designed eight PCR primer pairs (Supporting information Table S1)

170 using DNA sequences identified as being conserved (Murphy \& O'Brien 2007) between the

171 mtDNA genomes of the elephant (Hauf et al. 1999) and the aardvark (Arnason et al. 1999), or as

172 being conserved across published elephantid sequences. In addition to the PCR primers, within

173 each of the amplified regions we designed a set of additional sequencing primers to produce

174 forward or reverse sequences within each of the eight amplified segments (Supporting

175 information Table S1); together these sets generated overlapping sequences spanning the entire

176 mitochondrial genome, except for a repetitive non-coding region, containing a variable number

177 of tandem repeats (VNTR), that has also not been reported for most previously generated

178 proboscidean mitogenomes (Rogaev et al. 2006; Gilbert et al. 2007; Gilbert et al. 2008).

$179 \quad$ PCR and sequencing. Genomic DNA (ca. $50 \mathrm{ng}$ ) underwent amplification by PCR in a 25

$180 \mathrm{ul}$ reaction volume containing 1x GeneAmp PCR buffer II, $1.5 \mathrm{mM} \mathrm{MgCl}_{2}, 0.2 \mathrm{mM}$ each of the

181 four deoxyribonucleoside 5'-triphosphates (dATP, dCTP, dGTP, and dTTP), 0.04 unit/ul 
182 AmpliTaq-GOLD DNA Polymerase (Applied Biosystems Inc. [ABI]), and $0.4 \mathrm{uM}$ final

183 concentration of each oligonucleotide primer. PCR was run with an initial step of $95^{\circ} \mathrm{C}$ for $9: 45$

$184 \mathrm{~min}$; with cycles of $20 \mathrm{sec}$ at $94^{\circ} \mathrm{C}$; followed by $30 \mathrm{sec}$ at $60^{\circ} \mathrm{C}$ (first 3 cycles), $58^{\circ} \mathrm{C}$ (next 5

185 cycles), $56^{\circ} \mathrm{C}(5$ cycles $), 54^{\circ} \mathrm{C}(5$ cycles $), 52^{\circ} \mathrm{C}(5$ cycles $)$, or $50^{\circ} \mathrm{C}$ (final 22 cycles); followed by

1863 min extension at $72^{\circ} \mathrm{C}$; with a final extension after the last cycle of 7 min at $72^{\circ} \mathrm{C}$. PCR

187 products were enzyme-purified (Hanke \& Wink 1994) and sequenced using the BigDye

188 Terminator system (ABI). Extension products were purified using Sephadex G-50 (Amersham)

189 and resolved on an ABI 3700 DNA Sequencer, or on ABI 3730xl capillary systems. The

190 software Sequencher (Gene Codes Corporation) was used to trim and concatenate sequences and

191 confirm open reading frames. DNA sequences have been deposited in Genbank (accession

192 numbers: JN673263 and JN673264).

Avoidance of nuclear DNA sequences of mitochondrial origin (numts). Steps were taken

194 to minimize the possibility of amplifying numts. Primers were designed for conserved regions

195 (see above), which would minimize the risk of mismatches in the target mtDNA versus the

196 primers. Amplicons were several thousand bases in length, which would avoid those numts that

197 were shorter. Obvious indicators of numts were not observed: PCR did not produce multiple

198 bands; there were no sites that appeared heteroplasmic with secondary peaks; open reading

199 frames were present in all coding regions (see Supporting information Figure S1)--these would

200 have been disrupted in many numts; sequences overlapping between amplicons were identical in

201 the region of overlap, minimizing the possibility that primer mismatches had led to selective

202 amplification of numts over cytoplasmic mtDNA.

203 Sequence alignments. Three mtDNA genome sequences have been generated for S clade

204 African savanna elephants (Hauf et al. 1999; Rogaev et al. 2006; Murata et al. 2009), along with 
205 three for Asian elephants (Rogaev et al. 2006; Maikaew et al. 2007; Arnason et al. 2008),

206 nineteen for woolly mammoths (Krause et al. 2006; Rogaev et al. 2006; Gilbert et al. 2007;

207 Gilbert et al. 2008), and one Columbian mammoth mitogenome (Enk et al. 2011). The mtDNA

208 genome has also been sequenced for one proboscidean that is outside of the family Elephantidae,

209 the extinct American mastodon (Mammut americanum), which diverged from the lineage leading

210 to elephantids some 24-30 Mya (Rohland et al. 2007; Rasmussen \& Gutierrez 2009; Rohland et

211 al. 2010; Sanders et al. 2010). We retrieved complete mtDNA genomic sequences from

212 GenBank for seven elephantid individuals, including three African savanna elephants (GenBank:

213 DQ316069, NC000934, and AB443879) (Hauf et al. 1999; Rogaev et al. 2006; Murata et al.

214 2009), two Asian elephants (GenBank: NC005129.2 and AJ428946) (Rogaev et al. 2006;

215 Arnason et al. 2008) and two woolly mammoths (GenBank: NC007596.2 and EU153453)

216 (Krause et al. 2006; Gilbert et al. 2008). For some analyses, the mitogenomic sequence of an

217 American mastodon (Genbank: NC_009574) (Rohland et al. 2007) was also included. Published

218 genomes were aligned with the two newly generated African forest elephant sequences using the

219 software CLUSTALW 2.0 (Larkin et al. 2007) in EBI Web Services (McWilliam et al. 2009);

220 alignment output was visually inspected. The control region was excluded from analyses since it

221 is subject to greater saturation and is less reliable as a molecular clock relative than other

222 mtDNA regions (Ingman et al. 2000). Mismatches between the mtDNA genomes were

223 visualized using the software Geneious (Drummond et al. 2010).

224 Phylogenetic analyses. The Akaike Information Criterion (AIC) (Akaike 1974) was

225 implemented using the software Modeltest 3.06 (Posada \& Crandall 1998) to estimate the model

226 of DNA sequence evolution that best fit the data. This was found to be the Tamura-Nei model

227 with invariant site heterogeneity $(\operatorname{TrN}+\mathrm{I})$, with the following parameters: Base (base frequencies 
228 for $\mathrm{A}, \mathrm{C}$ and $\mathrm{G}$, with $\mathrm{T}$ inferred $)=(0.32860 .25230 .1358)$; Nst (number of substitution types

229 listed in a rate matrix) $=6$ (the number of unique substitution types for this model is 3 ; PAUP*

230 specifies this using a matrix with six values, four of which are identical); Rmat (rate matrix) $=$

$231(1.000038 .74251 .00001 .000055 .39861 .0000)$; Rates (distribution of rates at variable sites) $=$

232 equal; and Pinvar (proportion of invariant sites) $=0.7045$. These parameter values were used in

233 PAUP*4.0b10 (Swofford 2002) for Neighbor Joining (NJ), minimum evolution (ME), and

234 maximum likelihood (ML) phylogenetic methods. PAUP* was also used to infer a maximum

235 parsimony (MP) tree. For all phylogenetic methods, exhaustive searches were conducted.

236 Bootstrap resampling support was based on at least 100 replicates, in a full heuristic search with

237 starting trees obtained by random stepwise addition and tree bisection-reconnection branch

238 swapping for MP, ME and ML analyses. Bayesian phylogenetic inference was performed using

239 BEAST v1.5.4 software (Drummond \& Rambaut 2007), using the TN93 + I model of nucleotide

240 substitution, determined using the AIC (Akaike 1974). Maximum parsimony and maximum

241 likelihood analyses were also run using a partitioned genome (protein coding genes,

242 rRNA+tRNA genes, $\mathrm{H}$ strand and L strand genes), with the AIC (Akaike 1974) used to select the

243 model of evolution for each partition using Modeltest 3.06 (Posada \& Crandall 1998). A dataset

244 was run consisting of the entire mitogenome (excluding control region), but with each partition

245 allowed to follow a different model of evolution; each partition was also run separately

246 (Supporting information Text S1). In all cases inter-individual relationships were identical in all

247 phylogenies (Supporting information Text S1).

248 Molecular dating. Nodal divergence dates within the tree were estimated using the

249 software BEAST (Drummond \& Rambaut 2007), with fossil estimates used as prior dates for

250 calibration. We utilized an uncorrelated lognormal relaxed molecular clock model, which 
251 permits the rate of molecular substitution to be uncorrelated across the tree while incorporating

252 uncertainty in both tree topology and multiple fossil calibrations (Drummond et al. 2006). Fossil

253 calibration priors were incorporated into the clock model as a normal distribution representing

254 soft bounds $\left(5^{\text {th }}\right.$ and $95^{\text {th }}$ percentile corresponding to the minimum and maximum dates

255 respectively), since maximum fossil calibration dates incorporated uncertainty in the fossil

256 record (see below) (Forest 2009). We used a randomly generated starting tree. Posterior

257 distributions were obtained by Markov chain Monte Carlo (MCMC) sampling from a total of

$25810,000,000$ steps, with a discarded burn-in of 1,000,000; samples were drawn every 1,000

259 MCMC steps. Acceptable mixing and convergence to the stationary distribution were verified by

260 inspection and plotting of posterior samples. Effective sample size values were above 200 for all

261 parameters.

262 Two sets of prior date calibrations were used. The fossil calibration dates listed below

263 were applied to one dataset that included only elephantid sequences and calibration dates, and

264 then applied to a second dataset that included the American mastodon mitogenome as the

265 outgroup, and also used a mastodon-elephantid calibration in addition to the elephantid

266 calibration priors. Thus we performed a total of four analyses, two with only the elephantid

267 mitogenomes and two with the mastodon mitogenome included as the outgroup. The two sets of

268 prior dates used as calibrations were as follows:

269 (i) Our "broad" fossil calibration used the same fossil date range estimates as Rohland et

270 al. (2010), except that no prior date estimates were used for the divergence between African

271 elephant $\mathrm{F}$ and $\mathrm{S}$ mtDNA clades. Rohland et al. (2010) set the date range for the mastodon-

272 elephantid divergence as 24-30 Mya. The minimum was based on Sanders et al. (2010), who

273 mention the Rasmussen and Gutierrez (2009) report of fauna from the Eragaleit Beds of 
274 Lothidok as including the earliest definitively known mammutid, dated to between >24-27 Mya

275 (Rasmussen \& Gutierrez 2009). The maximum was also based on Sanders et al. (2010), who

276 mention a specimen from Chilga, Ethiopia (28-27 Ma) described as having mammutid features

277 superimposed on an otherwise palaeomastodont-type molar. To this and to other estimates of

278 maximum dates was added $2 \mathrm{My}$, to account for difficulty in recognizing differences during an

279 initial period of divergence, and to account for inadequate fossil sampling (Rohland et al. 2010).

280 The 2 My also approximates the maximum (1.8 My) of averages for elephantid species duration

281 that had been calculated by Maglio using six intervals of one million years of time, with 3 to 14

282 elephantid species present within each interval (Maglio 1973).

283 The range for the split between Loxodonta and Eurasian elephantids was set as 4.2-9 Mya

284 (we use "Eurasian" to refer to the clade including Asian elephants and woolly mammoths, while

285 recognizing that both Elephas and Mammuthus originated in Africa, and the woolly mammoth

286 range included North America) (Rohland et al. 2010). The minimum of 4.2 Mya was based on

287 Sanders et al. (2010), who list Elephas ekorensis, as "the most ancient unequivocal

288 representative of the genus Elephas" (Sanders et al. 2010). Among locations listed for this

289 species, the oldest is 5.0-4.2 Mya (Sanders et al. 2010). This minimum also assumes that

290 Loxodonta is paraphyletic. The maximum of 9 Mya does not assume that Loxodonta is

291 paraphyletic, and is based on the older bound (7 Mya) of the age estimate of 6-7 Mya for the

292 oldest Loxodonta fossil (Vignaud et al. 2002; Sanders et al. 2010), to which was added 2 Mya to

293 the maximum date for the reasons noted above (Rohland et al. 2010).

294 The range for the split between Elephas and Mammuthus was set at 3-8.5 Mya (Rohland

295 et al. 2010). Although the earliest reported fossil mammoth is Mammuthus subplanifrons,

296 Sanders et al. (2010) state that "in the absence of associated crania, however, there is no certainty 
297 that this species is a mammoth", and that M. africanavus is "the earliest unambiguous evidence

298 of the genus Mammuthus in Africa". In the associated table in Sanders et al. (2010), the latter

299 species is listed as mid-late Pliocene, with dated locales at 3.5-3.0 Mya (Sanders et al. 2010).

300 Rohland et al. therefore took 3 Mya as the minimum date for the Elephas-Mammuthus

301 divergence, which also assumes that Elephas is paraphyletic (Rohland et al. 2010). The

302 maximum date for the split did not assume paraphyly for the taxa. For the questionable $M$.

303 subplanifrons, the oldest date listed by Sanders et al. (2010) for any date range at a locale is 6

304 Mya, but there is an older "Elephas nawataensis" that dates to 4.2-6.5 Mya (Leakey \& Harris

305 2003). Although Sanders et al. (2010) suggest that this taxon may be mis-assigned to the genus

306 Elephas (Rohland et al. 2010), it was nonetheless included by Rohland et al. (2010) as the oldest

307 potential Elephas fossil identified in the literature; adding 2 Mya to the maximum date for the

308 reasons noted above provided the 8.5 Mya maximum (Rohland et al. 2010).

309 (ii) Our "narrow" fossil calibration range reduced the range of fossil date estimates in two

310 ways. First we removed from consideration Elephas nawataensis and Mammuthus subplanifrons

311 since Sanders et al. (2010) considered them to be potentially mis-assigned to their respective

312 genera. Second, we considered all genera to be monophyletic, consistent with several fossil

313 phylogenies (Maglio 1973; Shoshani \& Tassy 1996). This made the minimum date estimate for

314 the Loxodonta-Eurasian split equal to the lower bound of the age attributed to the oldest

315 Loxodonta fossil, or 6 Mya (Vignaud et al. 2002; Sanders et al. 2010), while the maximum date

316 for this divergence remained the same as for the "broad" fossil calibration. For the divergence

317 between Elephas and Mammuthus, the oldest fossil recognized by Sanders et al. (2010) as

318 definitely assigned to either genus is Elephas ekorensis. Among locations listed for this species,

319 the oldest is 4.2-5.0 Mya (Sanders et al. 2010). Thus the maximum date for the calibration of the 
320 split between Elephas and Mammuthus is 7.0 Mya (including two million years added for

321 reasons noted above). The assumption of monophyly would mean that this is also the fossil used

322 to set a date minimum for the Elephas-Mammuthus split, of 4.2 Mya (Sanders et al. 2010). The

323 "narrow" fossil calibration uses the same calibration dates as the "broad" fossil calibration for

324 the mastodon-elephantid split (for the dataset that included a mastodon mitogenome) (Rohland et

325 al. 2010). Like the "broad" fossil calibration, it used no prior estimate for the divergence

326 between $\mathrm{F}$ and $\mathrm{S}$ African elephant mtDNA clades.

328 Results

330 Forest elephant mitochondrial genomes

331 For the two forest elephants, we sequenced all 13 coding genes of the mtDNA genome,

332 along with the ribosomal RNAs and transfer RNAs, and part of the control region. We sequenced 33316,028 bp of mtDNA for forest elephant DS1534 and 16,156 bp of mtDNA for forest elephant

334 SL0001. We generated an alignment of the two forest elephant sequences with the mitochondrial 335 genomes of other elephantids, including all three previously sequenced mitogenomes for $\mathrm{S}$ clade

336 savanna elephants. We also aligned two Asian elephant and two woolly mammoth mtDNA

337 genomic sequences representing the deepest subdivisions present across the mtDNAs of those

338 species (Fernando et al. 2000; Fleischer et al. 2001; Krause et al. 2006; Gilbert et al. 2008;

339 Vidya et al. 2009). Although the mitogenome of a Columbian mammoth (Mammuthus columbi)

340 has been recently sequenced, we did not include it in our alignment because the Columbian

341 mammoth mitogenome had originated in the woolly mammoth lineage, and been transferred

342 between the two species through hybridization (Enk et al. 2011). 
A comparison of nucleotide differences across elephantid lineages is shown in Figure 1.

344 The top panel compares elephantid mitochondrial genomes to that of forest elephant DS1534,

345 while the bottom panel compares them to the reference savanna elephant S clade mitochondrial

346 genome (Hauf et al. 1999). The mitogenomes of the Loxodonta individuals shown are

347 representative of the deepest divergences present within the $\mathrm{F}$ and the $\mathrm{S}$ mtDNA clades

348 (Debruyne 2005; Roca et al. 2005; Lei et al. 2008; Murata et al. 2009). It is evident that the

349 differences between the two forest elephant $\mathrm{F}$ clade genomes (Figure 1, top panel) are greater

350 than the differences that exist across the savanna elephant $\mathrm{S}$ clade mitochondrial genomes

351 (Figure 1, bottom panel), consistent with an older coalescent date for F clade than for S clade

352 mitogenomes (see below).

\section{Phylogenetic analyses and molecular dating}

355 Phylogenetic relationships across lineages were inferred using maximum parsimony,

356 Neighbor-Joining, minimum evolution and maximum likelihood methods. All methods inferred

357 the same relationships among the elephantid mitogenomes. For all methods, all nodes were

358 supported with $100 \%$ bootstrap support. A Bayesian approach inferred the same relationships

359 across the phylogeny (Figure 2) as the other methods, with a Bayesian posterior probability of

3601.00 for all nodes. The results were consistent with previous molecular studies showing that

361 woolly mammoths are closer to Asian elephants than to African elephants (Krause et al. 2006;

362 Rogaev et al. 2006; Maikaew et al. 2007; Rohland et al. 2007; Murata et al. 2009; Rohland et al.

363 2010). Deep divergence was also inferred for the split between the $\mathrm{F}$ and $\mathrm{S}$ clade mtDNA

364 lineages in African elephants (Figure 2). 
To estimate divergence dates, we used two sets of fossil calibration dates for nodes on the

366 elephantid phylogeny (Table 1). Using the "broad" fossil calibration dates (see above, and Table

367 1), the divergence between Loxodonta and the other elephantids was estimated as occurring 6.06

368 (95\% CI 3.92-8.50) Mya, while the date for the Elephas-Mammuthus divergence was estimated

369 as 5.42 (95\% CI 3.40-7.62) Mya (Table 1). The divergence between the forest elephant F clade

370 mitochondrial genomes and the S clade savanna elephant genomes was estimated as 4.86 (95\%

371 CI 2.96-6.90) Mya, almost as deep as the split between Asian elephant and woolly mammoth

372 mitogenomes (Table 1). Using the "narrow" range of fossil calibration dates (see above, and

373 Table 1), the divergence between Loxodonta and the other elephantids was estimated as 6.81

374 (95\% CI 5.43-8.23) Mya (Table 1, Figure 2), while the Elephas-Mammuthus divergence was

375 estimated as 6.01 (95\% CI 4.71-7.17) Mya (Table 1, Figure 2). The divergence between African

376 elephant F and S clades was estimated as 5.51 (95\% CI 4.26-7.24) Mya, or about $92 \%$ of the

377 divergence time estimate for the mammoth-Asian elephant split (Table 1, Figure 2).

While it had been appropriate to use the mastodon as an outgroup for inferring

379 divergence dates among elephantid genera (Rohland et al. 2007; Roca 2008; Rohland et al.

380 2010), we thought that for inferring the divergence between the African elephant $\mathrm{F}$ and $\mathrm{S}$ clades,

381 fossil calibration dates for Loxodonta, Elephas and Mammuthus represented more recent and

382 thus more appropriate outgroups. Nonetheless, we considered and tested whether the addition of

383 the mastodon mitochondrial genome (Rohland et al. 2007) to the elephantid mitogenomic dataset

384 might have a great effect on divergence date estimates for the elephantids. Adding the mastodon

385 would have the benefit of polarizing the polymorphisms present across elephantids by

386 identifying potential ancestral character states for nucleotides. This could potentially improve the

387 accuracy of date estimates by rooting the crown of the elephantid tree. Yet when the mastodon 
mitogenome and calibration dates (Table 1) were included in the analyses, there was no effect on the relative estimates for the split between African elephant $\mathrm{F}$ and $\mathrm{S}$ clades as a proportion of the estimate for the split between Asian elephants and mammoth: the ratio was 0.92 when the mastodon was not included and 0.92 when the mastodon was included (Table 1), using the narrow fossil calibration dates. Thus rooting with the mastodon appears to have left divergence

393 ratios unchanged.

\section{Within-taxon coalescent dates}

The estimate for the F clade coalescent date was 2.57 (95\% CI 2.07-3.15) Mya for the

397 narrow fossil calibration priors when the mastodon mitogenome was included in the alignment

398 and the mastodon-elephantid calibration date was used along with fossil calibration dates for

399 elephantid genera (Table 1). This compared to 2.43 (95\% CI 1.68-3.38) Mya estimated using the

400 narrow fossil date priors as the F clade coalescent date with only elephantids used in the

401 alignment or as fossil calibrations (Table 1, Figure 2). Both estimates place the initial divergence

402 of crown group forest elephant mitogenomes as occurring near the start of the Pleistocene.

403 Our mtDNA coalescent estimates (Table 2) were compared to the within-taxon

404 coalescent estimates for nuclear loci determined by Rohland et al (2010). Our mtDNA coalescent

405 estimates were based on the most diverse mtDNA clades within each lineage. Our mtDNA

406 coalescent estimates are likely to accurately reflect basal within-lineage coalescent dates, and not

407 represent inadequate sampling, since sampling for all of the mtDNA lineages involved has been

408 quite extensive, involving dozens of locations and up to hundreds of individuals from across the

409 entire range of each taxon (Fernando et al. 2000; Fleischer et al. 2001; Krause et al. 2006;

410 Gilbert et al. 2008; Vidya et al. 2009; Ishida et al. 2011). Likewise, nuclear coalescent estimates 
411 are likely to be accurate since they had been calculated for each taxon using 375 loci (Rohland $e t$

412 al. 2010). In order to account for different assumptions or methods used to generate the

413 coalescent estimates, we normalized the intra-taxon coalescent dates as a percent of the

414 divergence estimated between Asian and African elephants (Table 2). In populations with

415 random progeny production and no sex difference in dispersal, mtDNA is expected to have an

416 effective population size (and hence coalescent date) one-fourth that of nuclear loci (Hedrick

417 2007). Yet for each elephantid taxon, the relative coalescent for mtDNA was much higher than

4180.25 , and in each case greater than the coalescent for nuclear DNA (Table 2). The most extreme

419 value was estimated for the woolly mammoth, for which the coalescent, standardized to the

420 Asian-African elephant divergence, was more than twice as old for mtDNA as for nuclear loci

421 (Table 2). We also compared the mtDNA coalescent to the coalescent of nuclear loci that were

422 two standard deviations above the mean nuclear coalescent estimate. The mtDNA:nuclear

423 coalescent ratio was much higher than 0.25 even when the coalescent for the mtDNA was

424 compared to the estimated coalescent for nuclear loci two standard deviations above the mean

425 (Table 2).

426

427 Discussion

428 For a number of elephantid taxa, mtDNA phylogeographic patterns appear to be 429 incongruent with nuclear genetic or morphological patterns (Debruyne 2005; Roca et al. 2005;

430 Roca et al. 2007; Lei et al. 2008; Lei et al. 2009; Enk et al. 2011; Ishida et al. 2011; Lei et al.

431 2011). One hypothesis has attributed this incongruence to sex differences in reproductive

432 competition and dispersal (Ishida et al. 2011). One prediction based on this hypothesis would be

433 that the ratio of mtDNA coalescent dates to nuclear coalescent dates should be much greater than 
434 the 0.25 expected in populations with random progeny production and no sex difference in

435 dispersal. In elephantids, both low female dispersal and high male variance in reproductive

436 success would tend to increase mtDNA effective population sizes relative to those of nuclear loci

437 (Hoelzer 1997; Hedrick 2007).

$438 \quad$ Using within-taxon coalescent estimates for nuclear markers estimated by Rohland et al.

439 (2010), we were able to determine that mtDNA coalescent dates were indeed older than those of

440 nuclear loci (Table 2). The coalescent data estimates for nuclear data are likely to accurately

441 reflect values for neutral loci in the nuclear genome since: (1) sequences had been randomly

442 generated, so were likely to be non-coding loci not under selection; and (2) repetitive elements

443 had been screened out, so that sequences across taxa were likely to represent orthologous and not

444 paralogous loci (Rohland et al. 2010). Elephantid mtDNA coalescent dates were very high

445 relative to nuclear coalescent dates even when the mtDNA coalescent was compared to the

446 estimated coalescent for nuclear loci that are two standard deviations above the mean coalescent

447 (Table 2). Thus our study provides support for the hypothesis that among elephantids coalescent

448 dates for mtDNA relative to nuclear DNA would be much older than the 0.25 expected in

449 populations with random progeny production and no sex difference in dispersal. The relatively

450 ancient mtDNA coalescent dates are consistent with lower female than male dispersal and

451 reproductive competition in elephantids, which would allow mtDNA genetic patterns to locally

452 persist even as male dispersal would disrupt any geographic subdivisions among nuclear alleles

453 (Wright 1943; Petit \& Excoffier 2009; Ishida et al. 2011).

454 The effects of sex differences in reproductive success on effective population size $\left(N_{e}\right)$

455 have been previously quantified (Hoelzer 1997; Hedrick 2007). As male effective population

456 sizes are reduced, $N_{e}$ drops for nuclear loci (Hoelzer 1997; Hedrick 2007). The effective 
457 population size becomes equal for autosomal, $\mathrm{X}$-linked and mtDNA loci when the male effective 458 population size is reduced to one-seventh that for females $\left(N_{e m}=N_{e f} / 7\right)$, i.e., when the effective 459 population size for males comprises one-eighth that of the total effective population size of males 460 and females combined (Hoelzer 1997; Hedrick 2007). Such a ratio could be consistent with the 461 lack of reproductive success documented for a large proportion of males in field studies of 462 savanna elephants (Hollister-Smith et al. 2007; Poole et al. 2011). For example, in a 22-year 463 study at Amboseli National Park, of 89 male elephants genotyped, 53 (60\%) were not found to 464 have sired a calf; while $30 \%$ of the 119 calves examined had been fathered by just three males 465 (Hollister-Smith et al. 2007). Male savanna elephants represent the extreme among mammals in 466 the extent to which high mating and paternity success occur late in life (Hollister-Smith et al. 467 2007). Age-specific paternity peaks at $45-53$ years of age (Hollister-Smith et al. 2007). Since 468 fewer than $10 \%$ of males are estimated to survive to age 50 (Poole et al. 2011), a high proportion 469 of males are not reproductively successful, and this high variance in reproductive success would 470 reduce the effective population size of nuclear markers (Hoelzer 1997; Hedrick 2007). Likewise, the lower degree of dispersal for female than male elephants would be 472 expected to increase the effective population size and coalescent of mtDNA relative to those of 473 nuclear loci (Wright 1943; Hoelzer 1997; Ishida et al. 2011). The interspecies transfer of F clade 474 mtDNA from forest to savanna elephant populations (Debruyne 2005; Roca et al. 2005; Ishida et 475 al. 2011) was also likely enhanced by low rates of female dispersal (Petit \& Excoffier 2009), 476 although we could disregard the effects of hybridization by considering only S clade coalescent 477 dates among savanna elephants. When dispersal rates for males are high while females exhibit 478 high matrilocality, the sex-biased dispersal can increase the effective population size of mtDNA 
479 relative to nuclear DNA even to the point of causing the coalescent of mtDNA to exceed that of

480 nuclear loci (Hoelzer 1997).

For both nuclear and mtDNA loci, coalescent estimates are low for the savanna elephant

482 (low diversity and effective population sizes relative to other taxa); high for the forest elephant

483 (highest diversity and effective population size); and intermediate for the Asian elephant

484 (intermediate diversity and effective population size) (Table 2) (Rohland et al. 2010). Thus the

485 rank order of genetic diversity for these three lineages was the same for mtDNA and nuclear

486 markers (Table 2). The savanna elephant has the lowest nuclear coalescent among the

487 elephantids (Rohland et al. 2010), and the mtDNA coalescent date for the savanna elephant $\mathrm{S}$

488 clade was also low relative to other taxa (Table 2). A number of authorities have suggested that

489 the geographic range and numbers of savanna elephants expanded towards the end of the

490 Pleistocene following the extinction of Elephas, which had been the predominant elephantid on

491 the African savannas until that time (Maglio 1973; Kingdon 1979; Sanders et al. 2010). The

492 relatively low coalescent values for both nuclear and S clade mtDNA in the savanna elephant

493 (relative to other taxa) could reflect this proposed Late Pleistocene founder effect for the savanna

494 elephant. Simulations and empirical data have shown that the interspecies transfer of alleles to a

495 species is expected to occur as its dispersing sex colonizes a region occupied by the other

496 species, with the other species contributing alleles transmitted by the non-dispersing sex to the

497 colonizing species (Currat et al. 2008; Petit \& Excoffier 2009). The transfer of F clade mtDNA

498 from forest to savanna elephants would be consistent with this expectation, if male savanna

499 elephants had expanded their range into formerly forest elephant habitats (Roca et al. 2005). The

500 F clade mtDNA sequences shared by forest and savanna species are quite similar (Eggert et al. 
501 2002; Roca et al. 2005), indicating that the interspecies transfer would be consistent with a Late

502 Pleistocene event (Murata et al. 2009).

503 For the woolly mammoth, the level of nuclear diversity was low and comparable to that

504 of savanna elephants. Yet the mtDNA coalescent date was much older for mammoths than for S

505 clade savanna elephants (Table 2). The low nuclear genetic diversity present in the woolly

506 mammoth could reflect high (relative to other species) levels of male reproductive competition

507 (Rohland et al. 2010). Male-male competition often also leads to the evolution of sexual size

508 dimorphism (Weckerly 1998), which is also observed in mammoths, with males about one meter

509 taller than females (Haynes 1991). The woolly mammoth mitochondrial coalescent seems

510 especially ancient relative to the nuclear coalescent estimate (Table 2), and the mammoth was

511 the only species for which the mtDNA coalescent estimate (Table 1, Figure 2) was more than

512 twice as old as those previously reported for nuclear loci (Rohland et al. 2010). This pattern may

513 reflect a more ancient widespread distribution for this species, its survival in multiple glacial

514 refugia, or the effects of the distribution of woolly mammoths across two continents that were

515 only intermittently connected during Pleistocene glacial cycles (Barnes et al. 2007; Debruyne et

516 al. 2008; Gilbert et al. 2008; Miller et al. 2008). These factors would be more likely to affect

517 mtDNA than nuclear genetic patterns among elephantids since (1) male-male competition would

518 lower effective population size for nuclear loci, reducing overall nuclear genetic diversity but not

519 affecting mtDNA diversity, and (2) male-mediated gene flow would tend to diminish nuclear

520 genetic differences across populations, while not affecting mtDNA differences across

521 populations.

522 As part of this study, we conducted the first full sequencing, to our knowledge, of

523 mitogenomes from forest elephants. These were, exclusive of the control region, 15,418 bp in 
524 length for DS1534, and 15,420 bp for SL0001. The size difference was due to an insertion of 2

525 bp in the 12S rRNA of SL0001, not found in any other proboscidean mitogenome. The

526 previously published savanna elephant S clade mitochondrial genome reference sequence was

527 15,418 bp in length (exclusive of control region) (Hauf et al. 1999). Since some savanna

528 elephants carry F clade mitochondrial genomes (Debruyne 2005; Roca et al. 2005), savanna

529 elephant nuclear genes involved in mitochondrial function would appear to be capable of

530 interacting with mtDNA-coded proteins whether these derive from $\mathrm{F}$ clade or $\mathrm{S}$ clade

531 mitochondrial genomes. Thus amino acid differences between forest $\mathrm{F}$ and previously sequenced

532 savanna S clade mtDNAs (Supporting information Figure S1) would not be expected to have led

533 to greatly altered protein function (McKenzie et al. 2003), although an alternative is possible.

534 Genetic markers carried only by the non-dispersing sex are expected to be less subject to the

535 effects of selection (Petit \& Excoffier 2009). This is somewhat paradoxical, given that the higher

536 effective population sizes among such markers (Wright 1943; Hoelzer 1997) would make fewer

537 mutations effectively neutral (Ohta 1973). However, limited gene flow can preclude selective

538 sweeps within species at loci carried only by the non-dispersing sex (Petit \& Excoffier 2009).

539 This may suggest that F clade mitogenomes in savanna elephants could persist even should they

540 lead to somewhat lower levels of fitness than S clade mitogenomes.

541 Our results also confirmed previous reports that mammoths are closer to living Asian

542 elephants than to African elephants (Krause et al. 2006; Rogaev et al. 2006; Maikaew et al.

543 2007; Rohland et al. 2007; Rohland et al. 2010). The split between Elephas and Mammuthus

544 occurred 6.0 (95\% CI 4.71-7.17) Mya, while Loxodonta F and S clades diverged 5.5 (95\% CI

545 4.26-7.24) Mya (Figure 2). Our estimate for the divergence of F clade and S clade African

546 elephant mitochondrial lineages is comparable to or somewhat older than previous estimates for 
547 the divergence between forest and savanna elephants (Roca et al. 2001; Roca et al. 2005;

548 Rohland et al. 2007; Murata et al. 2009), and suggests that the four major elephantid mtDNA

549 lineages were established around the end of the Miocene Epoch. In Africa during this period

550 there were cooler and more arid climates, changes in vegetation marked by a reduction in forests,

551 and shifts in habitat zones (Cerling et al. 1997; Cerling et al. 1998; Maley 2001; Griffin 2002;

552 Sepulchre et al. 2006; Bonnefille 2010; Otero et al. 2011). These changes may have driven the

553 evolution not only of elephantid lineages but also of other modern mammalian lineages,

554 including artiodactyls, perissodactyls, and primates including hominids (Cerling et al. 1998;

555 Folinsbee \& Brooks 2007; Roca 2008; Werdelin \& Sanders 2010).

556 The basal divergence represented by the two sequenced forest elephant mtDNA genomes

557 is estimated to have occurred 2.43 (95\% CI 1.68-3.38) Mya, around the beginning of the

558 Pleistocene Epoch 2.58 Mya (as recently defined to include the Gelasian Stage) (Gibbard \&

559 Head 2010). Divergence between humans and chimpanzees is comparable to the divergence

560 across elephantid taxa, yet the coalescent date for all living human mitogenomes is under

561200,000 years (Ingman et al. 2000). Previous studies have shown that mtDNA differences persist

562 in elephants within and between geographic regions even in the face of changes in habitats or in

563 nuclear genotypes (Roca et al. 2005; Roca \& O'Brien 2005; Roca et al. 2007; Ishida et al. 2011).

564 A close association of elephant mtDNA lineages with geographic region would be expected

565 since females do not typically migrate away from natal social groups. Since lack of female

566 dispersal increases the effective population size of mtDNA (Table 2), mtDNA lineages would be

567 removed by genetic drift much less rapidly than would nuclear diversity, so that geographic

568 partitions caused by ancient climate and habitat changes would be detectable in the mtDNA 
569 phylogeographic patterns long after the nuclear phylogeographic partitions had been erased by

570 male-mediate gene flow (Wright 1943; Hoelzer 1997; Petit \& Excoffier 2009).

571 The deepest subdivision within the forest elephant F clade mtDNA could reflect the

572 initial fragmentation of African tropical forest habitats during glacial cycles that began 2.5 Mya

573 (Figure 2) (Maley 2001). With the caveat that locations and definitions of Pleistocene refugia for

574 tropical forests remain controversial (Lowe et al. 2010), African tropical forest habitats are

575 believed to have repeatedly fragmented during glacial cycles into a set of discontinuous refugia

576 (Mayr \& Ohara 1986; Maley 2001; DeMenocal 2004). Forest elephant populations within

577 refugia may have been separated by intervening savanna habitats occupied by larger elephantid

578 species (Maglio 1973; Kingdon 1979; Sanders et al. 2010). The two forest elephant

579 mitogenomes sequenced were from an individual from the West African Guinean forest block

580 and an individual from the Central African Congolian forest block, although it is not clear

581 whether this deepest F clade subdivision was consequent to an east-west separation of the

582 tropical forest, as currently occurs (White 1983; Salzmann \& Hoelzmann 2005). Elephants

583 carrying mtDNA haplotypes from each of the two deepest subdivisions of the F clade are

584 currently present in populations in both West and Central Africa, an indication that some

585 migration of elephant females across the two forest blocks occurred subsequent to the basal F-

586 clade split (Debruyne et al. 2003). Also, given that gene flow in elephants is male-mediated and

587 that the long-distance dispersal of forest elephant nuclear alleles is well documented (Roca et al.

588 2001; Roca et al. 2005), the partitioning of mtDNA would not necessarily imply the

589 establishment or persistence of nuclear genetic differentiation between the two forest blocks

590 (Roca et al. 2007; Ishida et al. 2011). Nonetheless, since the ancient coalescent for mtDNA was

591 likely consequent to relatively low levels of competition and limited dispersal among matrilocal 
592 females, elephant mtDNA would be likely to persistently record a phylogeographic signature of

593 ancient climate transitions and habitat changes, such as occurred in Africa at the start of the

594 Pleistocene.

595

596 Acknowledgments:

598 We thank V. Buckley-Beason, F. Hussain, M. Malasky and Russ Hanson for technical or other 599 assistance. For elephant samples, we thank A. Turkalo and J. M. Fay; and A. Lécu at the Parc

600 Zoologique de Paris-Vincennes, France. We thank the government of the Central African

601 Republic for permission to collect samples. We thank M. Gadd and R. Ruggiero; funding was

602 provided by the U.S. Fish and Wildlife Service African Elephant Conservation Fund Grant no.

603 AFE-0554-96200-0-G051.

604

605 Conflict of interest:

606

607 The authors have declared that there is no conflict of interest.

608

609 References

610

611 Akaike H (1974) A new look at the statistical model identification. IEEE Transactions on Automatic Control 19, 716-723.

613 Archie EA, Hollister-Smith JA, Poole JH, et al. (2007) Behavioural inbreeding avoidance in wild $614 \quad$ African elephants. Mol Ecol 16, 4138-4148. 
615 Archie EA, Maldonado JE, Hollister-Smith JA, et al. (2008) Fine-scale population genetic structure in a fission-fusion society. Mol Ecol 17, 2666-2679.

617 Arnason U, Adegoke JA, Gullberg A, et al. (2008) Mitogenomic relationships of placental 618 mammals and molecular estimates of their divergences. Gene 421, 37-51.

619 Arnason U, Gullberg A, Janke A (1999) The mitochondrial DNA molecule of the aardvark, 620 Orycteropus afer, and the position of the Tubulidentata in the eutherian tree. Proc R Soc Lond B Biol Sci 266, 339-345.

622 Barnes I, Shapiro B, Lister A, et al. (2007) Genetic structure and extinction of the woolly mammoth, Mammuthus primigenius. Curr Biol 17, 1072-1075.

624 Barriel V, Thuet E, Tassy P (1999) Molecular phylogeny of Elephantidae. Extreme divergence of the extant forest African elephant. C R Acad Sci III 322, 447-454.

Bonnefille R (2010) Cenozoic vegetation, climate changes and hominid evolution in tropical Africa. Global and Planetary Change 72, 390-411.

Capelli C, MacPhee RD, Roca AL, et al. (2006) A nuclear DNA phylogeny of the woolly

Cerling TE, Ehleringer JR, Harris JM (1998) Carbon dioxide starvation, the development of C-4 mammoth (Mammuthus primigenius). Molecular Phylogenetics and Evolution 40, 620ecosystems, and mammalian evolution. Philosophical Transactions of the Royal Society

634 Cerling TE, Harris JM, MacFadden BJ, et al. (1997) Global vegetation change through the Miocene/Pliocene boundary. Nature 389, 153-158.

636 Comstock KE, Georgiadis N, Pecon-Slattery J, et al. (2002) Patterns of molecular genetic variation among African elephant populations. Mol Ecol 11, 2489-2498. 
638 Currat M, Ruedi M, Petit RJ, Excoffier L (2008) The hidden side of invasions: massive introgression by local genes. Evolution 62, 1908-1920.

Debruyne R (2005) A case study of apparent conflict between molecular phylogenies: the interrelationships of African elephants. Cladistics 21, 31-50.

Debruyne R, Chu G, King CE, et al. (2008) Out of America: ancient DNA evidence for a new world origin of late Quaternary woolly mammoths. Curr Biol 18, 1320-1326.

Debruyne R, Van Holt A, Barriel V, Tassy P (2003) Status of the so-called African pygmy

DeMenocal PB (2004) African climate change and faunal evolution during the PliocenePleistocene. Earth and Planetary Science Letters 220, 3-24.

Deraniyagala PEP (1955) Some Extinct Elephants, Their Relatives and the Two Living Species. Government Press (National Museum of Ceylon), Colombo. http://www.geneious.com.

653 Drummond AJ, Ho SYW, Phillips MJ, Rambaut A (2006) Relaxed phylogenetics and dating with confidence. PLoS Biol 4, e88.

655 Drummond AJ, Rambaut A (2007) BEAST: Bayesian evolutionary analysis by sampling trees. BMC Evol Biol 7, 214.

657 Eggert LS, Rasner CA, Woodruff DS (2002) The evolution and phylogeography of the African elephant inferred from mitochondrial DNA sequence and nuclear microsatellite markers. Proc R Soc Lond B Biol Sci 269, 1993-2006. 
660 Enk J, Devault A, Debruyne R, et al. (2011) Complete Columbian mammoth mitogenome suggests interbreeding with woolly mammoths. Genome Biol 12, R51.

662 Fernando P, Pfrender ME, Encalada SE, Lande R (2000) Mitochondrial DNA variation, phylogeography and population structure of the Asian elephant. Heredity 84, 362-372.

664 Fernando P, Vidya TN, Payne J, et al. (2003) DNA analysis indicates that Asian elephants are native to Borneo and are therefore a high priority for conservation. PLoS Biol 1, E6.

Fleischer RC, Perry EA, Muralidharan K, Stevens EE, Wemmer CM (2001) Phylogeography of the Asian elephant (Elephas maximus) based on mitochondrial DNA. Evolution Int J Org Evolution 55, 1882-1892.

Folinsbee KE, Brooks DR (2007) Miocene hominoid biogeography: pulses of dispersal and differentiation. Journal of Biogeography 34, 383-397.

Forest F (2009) Calibrating the Tree of Life: fossils, molecules and evolutionary timescales. Ann Bot 104, 789-794.

673 Georgiadis N, Bischof L, Templeton A, et al. (1994) Structure and history of African elephant populations: I. Eastern and southern Africa. J Heredity 85, 100-104.

Gibbard PL, Head MJ (2010) The newly-ratified definition of the Quaternary System/Period and

678 Gilbert MT, Drautz DI, Lesk AM, et al. (2008) Intraspecific phylogenetic analysis of Siberian redefinition of the Pleistocene Series/Epoch, and comparison of proposals advanced prior to formal ratification. Episodes 33, 152-158. woolly mammoths using complete mitochondrial genomes. Proc Natl Acad Sci U S A 105, 8327-8332.

Gilbert MT, Tomsho LP, Rendulic S, et al. (2007) Whole-genome shotgun sequencing of mitochondria from ancient hair shafts. Science 317, 1927-1930. 
683 Griffin DL (2002) Aridity and humidity: two aspects of the late Miocene climate of North Africa and the Mediterranean. Palaeogeography Palaeoclimatology Palaeoecology 182, 65-91.

685 Groves CP, Grubb P (2000) Do Loxodonta cyclotis and L. africana interbreed? Elephant 2, 4-7.

686 Grubb P, Groves CP, Dudley JP, Shoshani J (2000) Living African elephants belong to two species: Loxodonta africana (Blumenbach, 1797) and Loxodonta cyclotis (Matschie, 1900). Elephant 2, 1-4.

Hanke M, Wink M (1994) Direct DNA sequencing of PCR-amplified vector inserts following enzymatic degradation of primer and dNTPs. Biotechniques 17, 858-860.

Hauf J, Waddell PJ, Chalwatzis N, Joger U, Zimmermann FK (1999) The complete mitochondrial genome sequence of the African elephant (Loxodonta africana), phylogenetic relationships of Proboscidea to other mammals, and D-loop heteroplasmy. Zoology-Analysis of Complex Systems 102, 184-195. genetic drift. Evolution 61, 2750-2771.

Hoelzer GA (1997) Inferring phylogenies from mtDNA variation: Mitochondrial-gene trees versus nuclear-gene trees revisited. Evolution 51, 622-626.

Hollister-Smith JA, Poole JH, Archie EA, et al. (2007) Age, musth and paternity success in wild male African elephants, Loxodonta africana. Animal Behaviour 74, 287-296. the origin of modern humans. Nature 408, 708-713. 
705 Ishida Y, Oleksyk TK, Georgiadis NJ, et al. (2011) Reconciling apparent conflicts between 706 mitochondrial and nuclear phylogenies in African elephants. PLoS One 6, e20642.

707 Kingdon J (1979) East African Mammals: An Atlas of Evolution in Africa. Volume III Part B $708 \quad$ (Large Mammals). Academic Press, London.

709 Krause J, Dear PH, Pollack JL, et al. (2006) Multiplex amplification of the mammoth 710 mitochondrial genome and the evolution of Elephantidae. Nature 439, 724-727.

711 Larkin MA, Blackshields G, Brown NP, et al. (2007) Clustal W and Clustal X version 2.0.

712 Bioinformatics 23, 2947-2948.

713 Leakey MG, Harris JM (2003) Lothagam: the Dawn of Humanity in Eastern Africa. Columbia $714 \quad$ University Press, New York.

715 Lei R, Brenneman RA, Louis EE (2008) Genetic diversity in the North American captive African 716 elephant collection. Journal of Zoology 275, 252-267.

717 Lei R, Brenneman RA, Schmitt DL, Louis EE, Jr. (2009) Detection of cytonuclear genomic 718 dissociation in the North American captive African elephant collection. J Hered 100, $719 \quad 675-680$.

720 Lei R, Brenneman RA, Schmitt DL, Louis Jr. EE (2011) Genetic diversity in North American $721 \quad$ captive Asian elephants. Journal of Zoology, published online 26 Sep 2011.

722 Lisiecki LE, Raymo ME (2005) A Pliocene-Pleistocene stack of 57 globally distributed benthic 723 delta O-18 records. Paleoceanography 20.

724 Lowe AJ, Harris D, Dormontt E, Dawson IK (2010) Testing putative African tropical forest 725 refugia using chloroplast and nuclear DNA phylogeography. Tropical Plant Biol 3, 50$726 \quad 58$. 
727 Maglio VJ (1973) Origin and evolution of the Elephantidae. Trans. Am. Phil. Soc. Philad., New $728 \quad$ Series $\mathbf{6 3}, 1-149$.

729 Maikaew U, Wajjwalku W, Siripholwat V, et al. (2007) [The study of nucleotides arrangement 730 of mitochondrial DNA in Thai elephants]. KKU Vet. J. 17, 11-21.

731 Maley J (2001) The impact of arid phases on the African rain forest through geological history.

Mayr E (1963) Animal Species and Evolution. Harvard University Press, Cambridge, MA.

Mayr E, Ohara RJ (1986) The biogeographic evidence supporting the Pleistocene forest refuge In: African Rain Forest Ecology and Conservation: An Interdisciplinary Perspective (eds. Weber W, White LJT, Vedder A, Naughton-Treves L). Yale University Press, New Haven [Conn.]. hypothesis. Evolution 40, 55-67.

McKenzie M, Chiotis M, Pinkert CA, Trounce IA (2003) Functional respiratory chain analyses in murid xenomitochondrial cybrids expose coevolutionary constraints of cytochrome b and nuclear subunits of complex III. Mol Biol Evol 20, 1117-1124.

McWilliam H, Valentin F, Goujon M, et al. (2009) Web services at the European Bioinformatics Institute-2009. Nucleic Acids Research 37, W6-W10.

743 Meier R, Wheeler Q (2000) Species Concepts and Phylogenetic Theory: A Debate. Columbia

$744 \quad$ University Press, New York.

745 Miller W, Drautz DI, Ratan A, et al. (2008) Sequencing the nuclear genome of the extinct woolly mammoth. Nature 456, 387-390.

747 Murata Y, Yonezawa T, Kihara I, et al. (2009) Chronology of the extant African elephant species and case study of the species identification of the small African elephant with the molecular phylogenetic method. Gene 441, 176-186. 
750 Murphy WJ, O'Brien SJ (2007) Designing and optimizing comparative anchor primers for

751 comparative gene mapping and phylogenetic inference. Nat Protoc 2, 3022-3030.

752 Ohta T (1973) Slightly deleterious mutant substitutions in evolution. Nature 246, 96-98.

753 Otero O, Lecuyer C, Fourel F, et al. (2011) Freshwater fish delta O-18 indicates a Messinian

$754 \quad$ change of the precipitation regime in Central Africa. Geology 39, 435-438.

755 Petit RJ, Excoffier L (2009) Gene flow and species delimitation. Trends Ecol Evol 24, 386-393.

756 Poole JH, Lee PC, Moss C (2011) Longevity, competition and musth: a long-term perspective on

757 male reproduction. In: The Amboseli Elephants: A Long-Term Perspective on a Long-

758 Lived Mammal (eds. Moss C, Lee PC, Croze H). University of Chicago Press, Chicago.

759 Posada D, Crandall KA (1998) MODELTEST: testing the model of DNA substitution.

$760 \quad$ Bioinformatics 14, 817-818.

761 Rasmussen DT, Gutierrez M (2009) A mammalian fauna from the Late Oligocene of northwestern Kenya. Palaeontographica Abteilung a-Palaozoologie-Stratigraphie 288,

764 Rasmussen HB, Okello JBA, Wittemyer G, et al. (2008) Age- and tactic-related paternity success in male African elephants. Behavioral Ecology 19, 9-15.

Roca AL (2008) The mastodon mitochondrial genome: a mammoth accomplishment. Trends Genet 24, 49-52.

768 Roca AL, Georgiadis N, O'Brien SJ (2005) Cytonuclear genomic dissociation in African elephant species. Nat Genet 37, 96-100.

770 Roca AL, Georgiadis N, O'Brien SJ (2007) Cyto-nuclear genomic dissociation and the African elephant species question. Quaternary International 169-170, 4-16. 
772 Roca AL, Georgiadis N, Pecon-Slattery J, O'Brien SJ (2001) Genetic evidence for two species of 773 elephant in Africa. Science 293, 1473-1477.

774 Roca AL, O'Brien SJ (2005) Genomic inferences from Afrotheria and the evolution of elephants.

$775 \quad$ Curr Opin Genet Dev 15, 652-659.

776 Rogaev EI, Moliaka YK, Malyarchuk BA, et al. (2006) Complete mitochondrial genome and 777 phylogeny of Pleistocene mammoth Mammuthus primigenius. PLoS Biol 4, e73.

778 Rohland N, Malaspinas AS, Pollack JL, et al. (2007) Proboscidean mitogenomics: chronology and mode of elephant evolution using mastodon as outgroup. PLoS Biol 5, e207.

780 Rohland N, Reich D, Mallick S, et al. (2010) Genomic DNA sequences from mastodon and woolly mammoth reveal deep speciation of forest and savanna elephants. PLoS Biol $\mathbf{8}$, e1000564.

783 Salzmann U, Hoelzmann P (2005) The Dahomey Gap: an abrupt climatically induced rain forest fragmentation in West Africa during the late Holocene. Holocene 15, 190-199.

785 Sambrook J, Fritsch EF, Maniatis T (1989) Molecular Cloning: A Laboratory Manual. 2nd edn. Cold Spring Harbor Laboratory Press, Cold Spring Harbor, N.Y.

787 Sanders WJ, Gheerbrant E, Harris JM, Saegusa H, Delmer C (2010) Proboscidea. In: Cenozoic Mammals of Africa (eds. Werdelin L, Sanders WJ). University of California Press,

790 Sepulchre P, Ramstein G, Fluteau F, et al. (2006) Tectonic uplift and Eastern Africa aridification. Science 313, 1419-1423.

792 Shoshani J, Tassy P (1996) The Proboscidea: Evolution and Palaeoecology of Elephants and 793 their Relatives. Oxford University Press, Oxford ; New York. 
794 Swofford DL (2002) PAUP*: Phylogenetic Analysis Using Parsimony (*and Other Methods).

795 Version 4.0b10. Sinauer, Sunderland, MA.

796 Vidya TN, Sukumar R, Melnick DJ (2009) Range-wide mtDNA phylogeography yields insights

797 into the origins of Asian elephants. Proc Biol Sci 276, 893-902.

798 Vignaud P, Duringer P, Mackaye HT, et al. (2002) Geology and palaeontology of the Upper

799 Miocene Toros-Menalla hominid locality, Chad. Nature 418, 152-155.

800 Weckerly FW (1998) Sexual-size dimorphism: Influence of mass and mating systems in the most

801 dimorphic mammals. Journal of Mammalogy 79, 33-52.

802 Werdelin L, Sanders WJ (2010) Cenozoic Mammals of Africa. University of California Press,

803 Berkeley.

804 White F (1983) The Vegetation of Africa. UNESCO, Paris.

805 Wright S (1943) Isolation by distance. Genetics 28, 114-138.

806

Data Accessibility:

808

809 DNA sequences: Genbank accession numbers Loxodonta cyclotis JN673263 and JN673264.

812 Figure 1. Sequence differences found among the mitochondrial genomes of elephantids

813 with respect to African forest (LCY) or savanna (LAF) elephants. Nucleotide comparisons

814 between a reference genome and those of other elephantids are shown by horizontal bars in the

815 top and bottom panels, with nucleotide matches represented by the white background,

816 substitutions indicated by black vertical lines, and indels by horizontal lines or dots. Top panel: 
817 comparison of elephantid mtDNA genomic sequences to reference forest elephant DS1534. Deep

818 within-species differences are evident in the comparison to SL0001. Center panel: nucleotide

819 positions within the mtDNA genome and locations of mtDNA regions. The D-loop was excluded

820 due to saturation of sites. Lower panel: comparison of elephantid mtDNA genomic sequences to

821 reference savanna elephant NC_000934 (Hauf et al. 1999). Note that differences present across

822 the savanna elephant S clade (DQ316069 and AB443879) are not as great as the differences

823 across forest elephants shown in the top panel, reflecting the more recent coalescent date for

824 savanna elephant S clade genomes. Panels were drawn using Geneious v5.4 (Drummond et al.

825 2010). Abbreviations: LCY: Loxodonta cyclotis, African forest elephant; LAF: Loxodonta

826 africana, African savanna elephant; MPR: Mammuthus primigenius, woolly mammoth; EMA:

827 Elephas maximus, Asian elephant.

829 Figure 2. Maximum clade probability tree inferred from the alignment of elephantid

830 mitochondrial genome sequences. The software BEAST (Drummond \& Rambaut 2007) was

831 used to generate the tree, which is displayed as a chronogram. Shaded bars indicate the $95 \%$

832 highest posterior density as determined using two sets of calibrations. The top bar used the

833 broadest range of calibrations based on fossil estimates (Rohland et al. 2010; Sanders et al.

834 2010); the lower bar relied on "narrow" set of calibration dates that excluded fossils identified as

835 being of questionable assignment to genera by Sanders et al. (2010), and assumed the monophyly

836 of elephantid genera (Maglio 1973; Shoshani \& Tassy 1996; Rohland et al. 2010). The tree

837 shown was based on the narrow fossil calibrations. The divergence date between forest elephant

838 (F clade) and savanna elephant (S clade) mtDNA genomes was comparable to the divergence

839 between Asian elephant and mammoth mtDNA genomes. For all four lineages, the two or three 
840 genomes shown encompass the most basal within-lineage divergences; these indicate that forest

841 elephants have the deepest within-lineage crown-group coalescent date, while S clade savanna

842 elephants have the most recent mtDNA coalescent date. The posterior probability was 1.00 for

843 all nodes (leftmost number). The same relationships across mitogenomes were inferred in

844 PAUP* (Swofford 2002) using maximum parsimony (MP), Neighbor Joining (NJ), minimum

845 evolution (ME) and maximum likelihood (ML) methods (not shown). Bootstrap support was

$846100 \%$ for each method at each node (shown respectively from left to right, from the second

847 number at each node, for MP, NJ, ME and ML). Tree scores were: MP, 1611 variable sites, 1106

848 parsimony informative, 1 tree, length $=1867, \mathrm{CI}=0.876, \mathrm{RC}=0.764$; $\mathrm{ME}, 1$ tree, score $=0.12588$;

849 ML, 1 tree, - -ln likelihood=31041.20336. A time scale and geological epochs are shown below

850 the phylogeny. Africa in the late Miocene experienced drier climates and the retreat of forests

851 (Bonnefille 2010), and shifts in habitat zones during the Messinian (Griffin 2002). Shown also is

852 a Pliocene-Pleistocene stack of 57 globally distributed benthic $\delta^{18} \mathrm{O}$ records, which measure

853 global ice volume and deep ocean temperature (lower values reflect lower temperatures), and

854 which illustrate the initiation of glacial cycles at the start of the Pleistocene (Lisiecki \& Raymo 855 2005). 







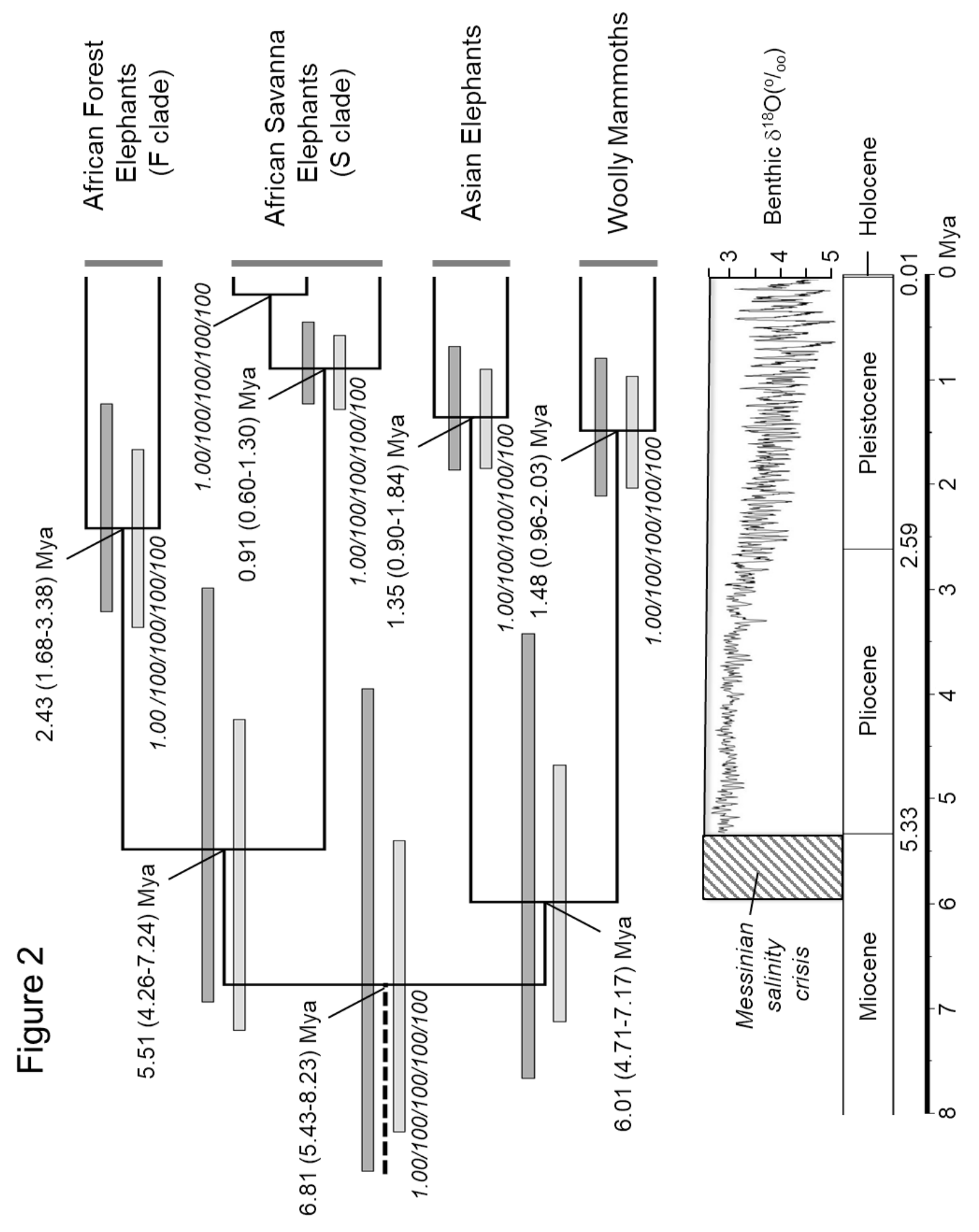


This is an Accepted Manuscript of an article published by Wiley in Molecular Ecology, available online: https://doi.org/10.1111/j.1365-294X.2012.05461.x.

Table 1. Proboscidean fossil calibration dates and divergence time estimates, in millions of years

\begin{tabular}{|c|c|c|}
\hline Calibration used: & $\begin{array}{c}\text { Narrow Fossil }{ }^{1} \\
\text { Median }(95 \% \mathrm{Cl})\end{array}$ & $\begin{array}{c}\text { Broad Fossil }{ }^{2} \\
\text { Median }(95 \% \mathrm{Cl})\end{array}$ \\
\hline \multicolumn{3}{|c|}{ Prior dates used to estimate divergence times } \\
\hline Elephantid-Mastodon & $27.0(24-30)$ & $27.0(24-30)$ \\
\hline Loxodonta-Eurasian & $7.5(6-9)$ & $6.6(4.2-9)$ \\
\hline Asian-Mammoth & $5.6(4.2-7)$ & $5.75(3-8.5)$ \\
\hline Forest-Savanna & Not used & Not used \\
\hline Monophyly of genera? & yes & no \\
\hline
\end{tabular}

Divergence dates estimated using elephantid mitogenomes and priors only

$\begin{array}{lcr}\text { Loxodonta-Eurasian } & 6.81(5.43-8.23) & 6.06(3.92-8.50) \\ \text { Asian-Mammoth } & 6.01(4.71-7.17) & 5.42(3.40-7.62) \\ \text { F clade-S clade } & 5.51(4.26-7.24) & 4.86(2.96-6.90) \\ \quad \text { Within-taxon coalescent } & \text { dates estimated using elephantid mitogenom } \\ \text { Woolly Mammoth } & 1.48(0.96-2.03) & 1.33(0.78-2.08) \\ \text { Asian Elephant } & 1.35(0.90-1.84) & 1.20(0.66-1.84) \\ \text { African Savanna S clade } & 0.91(0.60-1.30) & 0.80(0.45-1.23) \\ \text { African Forest F clade } & 2.43(1.68-3.38) & 2.13(1.22-3.19)\end{array}$

Divergence dates estimated by elephantid and mastodon mitogenomes and priors

$\begin{array}{lcc}\text { Elephantid-Mastodon } & 25.35(23.60-26.89) & 26.19(22.48-29.24) \\ \text { Loxodonta-Eurasian } & 7.13(6.29-8.06) & 7.43(6.06-8.61) \\ \text { Asian-Mammoth } & 6.35(5.57-7.22) & 6.65(5.39-7.80) \\ \text { F clade-S clade } & 5.83(4.94-6.71) & 6.06(4.85-7.17)\end{array}$

Within-taxon coalescent dates estimated by elephantid and mastodon mitogenomes and priors

Woolly Mammoth

$1.62(1.28-2.05) \quad 1.70(1.20-2.17)$

Asian Elephant

$1.33(1.00-1.65) \quad 1.41(1.07-1.79)$

African Savanna S clade

$0.76(0.57-0.99) \quad 0.81(0.60-1.11)$

African Forest F clade

$2.57(2.07-3.15) \quad 2.69(1.99-3.39)$

African-Eurasian ratio ${ }^{3}$ (elephantid priors only)

$0.92 \quad 0.90$

African-Eurasian ratio ${ }^{4}$

(mastodon+elephantid

$0.92 \quad 0.91$

priors)

F-S clade ratio ${ }^{5}$ (with and without mastodon)

$\begin{array}{ll}1.06 & 1.25\end{array}$

Footnotes:

${ }^{1}$ Narrow date priors are fossil dates described in Rohland et al. (2010), based on Sanders et al. (2010), with questionable taxa removed and assuming monophyly

${ }^{2}$ Broad date priors are fossil dates described in Rohland et al. (2010), based on Sanders et al. (2010)

3Ratio: F clade-S clade to Asian-Mammoth divergence, estimated using only elephantid mitogenomes and priors

${ }^{4}$ Ratio: F clade-S clade to Asian-Mammoth divergence, estimated using elephantid and mastodon mitogenomes and priors

${ }^{5}$ Ratio: F clade-S clade divergence calculated using mastodon and elephantid mitogenomes

and priors vs using only elephantid mitogenomes and priors 
This is an Accepted Manuscript of an article published by Wiley in Molecular Ecology, available online: https://doi.org/10.1111/j.1365-294X.2012.05461.x.

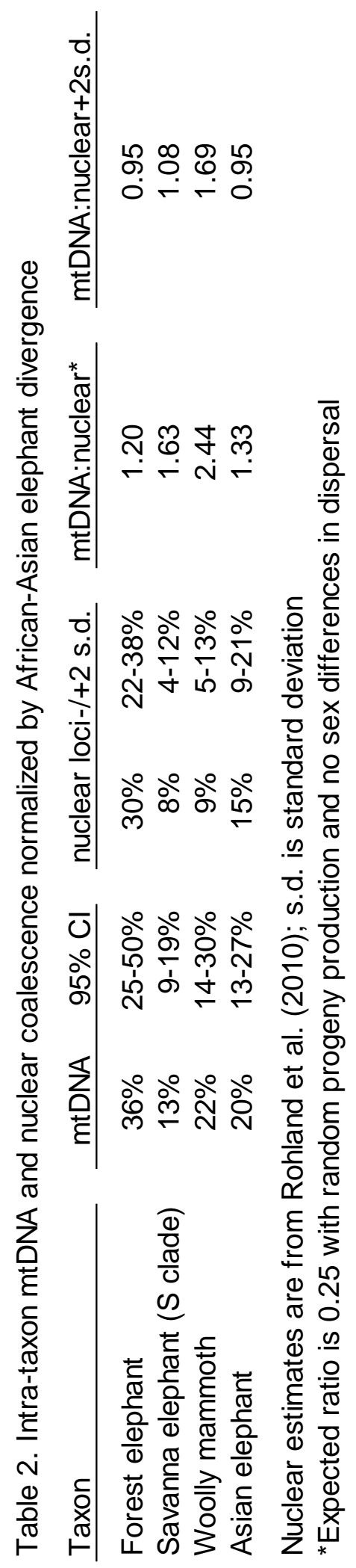




\title{
Forest elephant mitochondrial genomes reveal that elephantid diversification in Africa tracked climate transitions
}

\author{
Adam L. Brandt ${ }^{1}$, Yasuko Ishida ${ }^{1}$, Nicholas J. Georgiadis ${ }^{2}$ and Alfred L. Roca ${ }^{1,3 *}$ \\ ${ }^{1}$ Department of Animal Sciences, University of Illinois at Urbana-Champaign, Urbana, IL 61801 \\ USA \\ ${ }^{2}$ Director, Bole and Klingenstein Foundation, Cody, WY 82414 USA \\ ${ }^{3}$ Institute for Genomic Biology, University of Illinois at Urbana-Champaign, Urbana, IL 61801 \\ USA
}

*Corresponding Author

SUPPORTING INFORMATION 


\section{Supporting Information Text S1. Phylogenetic analysis of partitioned mitogenomes.}

The full mitochondrial genome was partitioned into protein coding genes, rRNA+tRNA genes, H-strand genes and L-strand genes. The Akaike Information Criterion (AIC) (Akaike 1974) was implemented using the software Modeltest 3.06 (Posada \& Crandall 1998) to estimate the model of DNA sequence evolution that best fit each partition. AIC parameter values were used in PAUP*4.0b10 (Swofford 2002) for maximum likelihood (ML) phylogenetic analysis, with each partition run individually. Although models cannot be applied to the maximum parsimony tree, PAUP* was also used to infer a maximum parsimony (MP) tree for each of the datasets. For both ML and MP methods, exhaustive searches were conducted and yielded topologies consistent with un-partitioned data; in each case relationships among the individuals on the tree were the same. Model parameters and tree statistics are as follows:

The model of evolution determined by Modeltest is listed here for each partition as follows: "Base" indicates the base frequencies for A, C and G, with T inferred (bases are "equal" when the model assumes frequencies are the same). "Nst" lists the number of substitution types listed in a rate matrix; the number of unique types may be inferred. This is followed by "Rmat", the rate matrix. "Rates" indicates the distribution of rates at variable sites. "Pinvar" indicates the proportion of invariant sites:

For Protein coding genes: Tamura-Nei model with invariant site heterogeneity $(\operatorname{TrN}+\mathrm{I})$. Base $=(0.32780 .25440 .1307) \mathrm{Nst}=6\left(\right.$ unique Nst=3) $\mathrm{Rmat}=\left(\begin{array}{l}1.000039 .86901 .00001 .0000 \\ 0\end{array}\right.$ 54.9781 1.0000) Rates=equal Pinvar $=0.6883$. 
For rRNA and tRNA genes: Tamura-Nei model with invariant site heterogeneity $(\mathrm{TrN}+\mathrm{I})$. Base $=(0.35720 .2134$ 0.1493) Nst=6 (unique Nst=3) Rmat=(1.0000 25.9384 1.00001 .0000 56.4363 1.0000) Rates=equal Pinvar=0.8094

For H-strand: Tamura-Nei model with invariant site heterogeneity ( $\mathrm{TrN}+\mathrm{I})$. Base $=\left(\begin{array}{l}0.3271 \\ 0.2495\end{array}\right.$ 0.1336) $\mathrm{Nst}=6$ (unique Nst=3) Rmat=(1.0000 38.18811 .00001 .0000 53.5181 1.0000) Rates=equal Pinvar=0.6923

For L-strand: Tamura-Nei model with invariant site heterogeneity ( $\mathrm{TrN}+\mathrm{I})$.

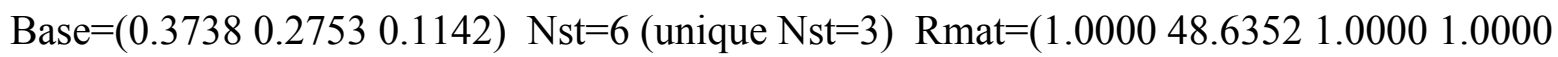
89.7056 1.0000) Rates $=$ equal Pinvar $=0.7637$

Tree scores were as follows for each partition:

For protein coding genes: MP, 1410 variable sites, 975 parsimony informative, 1 tree, Length $=1632, \mathrm{CI}=0.878, \mathrm{RI}=0.875, \mathrm{RC}=0.768 ; \mathrm{ML}, 1$ tree, - ln likelihood=23929.12496.

For rRNA and tRNA genes: MP, 186 variable sites, 119 parsimony informative, 1 tree, Length $=216, \mathrm{CI}=0.861, \mathrm{RI}=0.859, \mathrm{RC}=0.740 ; \mathrm{ML}, 1$ tree, - ln likelihood $=6610.969593$. For H-strand: MP, 1505 variable sites, 1035 parsimony informative, 1 tree, Length $=1739, \mathrm{CI}=0.879, \mathrm{RI}=0.876, \mathrm{RC}=0.771 ; \mathrm{ML}, 1$ tree, - ln likelihood=28913.41203.

For L-strand: MP, 93 variable sites, 61 parsimony informative, 1 tree, Length=111, $\mathrm{CI}=0.838, \mathrm{RI}=0.835, \mathrm{RC}=0.699 ; \mathrm{ML}, 1$ tree, $-\ln$ likelihood=1924.21973.

The AIC parameter values were also used in BEAST (Drummond \& Rambaut 2007) for Bayesian posterior probability estimation, using a dataset consisting of the entire mitogenome (excluding control region), but with each partition allowed to follow a different model of 
evolution. Additional settings included the use of an uncorrelated lognormal relaxed molecular clock model and a randomly generated starting tree; all other parameters remained as default settings. Posterior distributions were obtained by Markov chain Monte Carlo (MCMC) sampling from a total of 10,000,000 steps, with a discarded burn-in of 1,000,000; samples were drawn every 1,000 MCMC steps. Acceptable mixing and convergence to the stationary distribution were verified by inspection and plotting of posterior samples. Effective sample size values were above 200 for parameters. Posterior probabilities were as follows:

\begin{tabular}{|c|c|}
\hline Node & Posterior Probability \\
\hline Loxodonta-Eurasian clade & 1.00 \\
\hline Asian - mammoth clade & 1.00 \\
\hline F mtDNA - S mtDNA clade & 1.00 \\
\hline Woolly mammoth crown group & 1.00 \\
\hline Asian elephant crown group & 1.00 \\
\hline African savanna $S$ clade & 1.00 \\
\hline African forest $\mathrm{F}$ clade & 1.00 \\
\hline
\end{tabular}

\section{References}

Akaike H (1974) A new look at the statistical model identification. Automatic Control, IEEE Transactions on 19, 716-723.

Drummond AJ \& Rambaut A (2007) BEAST: Bayesian evolutionary analysis by sampling trees. BMC Evol Biol 7, 214. 
Posada D \& Crandall KA (1998) MODELTEST: testing the model of DNA substitution. Bioinformatics 14, 817-818.

Swofford DL (2002) PAUP*: Phylogenetic Analysis Using Parsimony (*and Other Methods). Version 4.0b10. Sinauer, Sunderland, MA. 
Table S1. Oligonucleotide primers used for PCR and sequencing of elephant mitochondrial DNA (mtDNA)

\begin{tabular}{|c|c|c|c|c|}
\hline Primer name & Primer function & Primer sequence & mtDNA regions (full or partial): & PCR amplicon size (bp) \\
\hline \multicolumn{5}{|l|}{ Set 1} \\
\hline mtAll-F1 & PCR, Sequencing & CCCCCACGGGAWACAGCAG & \multirow{8}{*}{$\begin{array}{l}\text { 12S ribosomal RNA } \\
\text { tRNA-Val } \\
16 S \text { ribosomal RNA }\end{array}$} & \multirow[t]{8}{*}{2282} \\
\hline mtAll-F12 & Sequencing & AAGTGAGCTTAATCATAACCCATGA & & \\
\hline mtAll-F13 & Sequencing & AATAGAGATAGTACCGCAAGGGAAT & & \\
\hline mtAll-F14 & Sequencing & CACTGCCTGCCCAGTGACCAAG & & \\
\hline mtAll-R1 & PCR, Sequencing & GTCTGAACTCAGATCACGTAGGACT & & \\
\hline mtAll-R11 & Sequencing & CGGTCTGAACTCAGATCACGTAG & & \\
\hline mtAll-R12 & Sequencing & GAGACAGTTAAACCCTCGTGTG & & \\
\hline mtAll-R13 & Sequencing & TGAATTTACTCGCTAATCAAGGTTGT & & \\
\hline \multicolumn{5}{|c|}{ ע } \\
\hline mtAll-F2 & PCR, Sequencing & GACCTCGATGTTGGATCAGG & \multirow{12}{*}{$\begin{array}{l}\text { 16S ribosomal RNA } \\
\text { tRNA-Leu } \\
\text { NADH dehydrogenase subunit } 1 \text { (ND1) } \\
\text { tRNA-lle } \\
\text { tRNA-GIn } \\
\text { tRNA-Met } \\
\text { NADH dehydrogenase subunit } 2(N D 2) \\
\text { tRNA-Trp } \\
\text { tRNA-Ala }\end{array}$} & 2821 \\
\hline mtAll-F21 & Sequencing & CCCCAATTCTAGCCCTAACC & & \\
\hline mtAll-F22 & Sequencing & GAAACTAACCGAGCCCCATT & & \\
\hline mtAll-F23 & Sequencing & CGAACCTAAACTCGAGAATTCAA & & \\
\hline mtAll-F24b & Sequencing & CACCTACACCAACACCAACCT & & \\
\hline mtAll-R2 & PCR, Sequencing & TGCAAATTCRAAGAAGCAG & & \\
\hline mtAll-R21 & Sequencing & AAATTATTGGGGTTAGGGGAAG & & \\
\hline mtAll-R22 & Sequencing & TGTTTTGTTTCATATTAGAGTTAGGG & & \\
\hline mtAll-R23 & Sequencing & ATAATGGTGCTAATTTTTGTCAGGT & & \\
\hline mtAll-R24 & Sequencing & AGTTGGTCGTATCGGAATCG & & \\
\hline mtAll-R25 & Sequencing & TTTGATGAGGTTTGTGGGTAGA & & \\
\hline mtAll-R26 & Sequencing & TTCGGGGTATGGGCCCGATAGC & & \\
\hline Set 3 & & & & \\
\hline mtAll-F3 & PCR, Sequencing & AACTGGCTTCAATCTACTTCTCC & tRNA-Cys & 2401 \\
\hline mtAll-F31 & Sequencing & TAATTGGAGGCTTTGGAAACTG & tRNA-Tyr & \\
\hline mtAll-F32 & Sequencing & TCCCAGTTCTAGCAGCAGGTAT & Cytochrome c oxidase subunit I (COX1) & \\
\hline mtAll-F33 & Sequencing & CTACCCTTCATGGCGGTAA & tRNA-Ser & \\
\hline mtAll-F34 & Sequencing & CCTGCAGGAGGAGGAGACCCAAT & & \\
\hline mtAll-R3 & PCR, Sequencing & GGAATTGCRTCKGTTTTTAGACCT & tRNA-Asp & \\
\hline mtAll-R31 & Sequencing & ACAAGACTAAGGAGCTAATAAGGAAAA & Cytochrome c oxidase subunit II (COX2) & \\
\hline mtAll-R32 & Sequencing & TTAGAAGCAAATGCCTCTCAAATTAT & & \\
\hline mtAll-R33 & Sequencing & GTCATGTAGGACAATGTCTAGTGAAG & & \\
\hline mtAll-R34b & Sequencing & TTGAGATTGCGATCCGTTAAT & & \\
\hline mtAll-R36 & Sequencing & AAGCTCGAGTGTCAACGTCTATGCC & & \\
\hline Set 4 & & & & \\
\hline mtAll-F4 & PCR, Sequencing & CACTTCCAYGACCAYACCCTAATAAT & Cytochrome c oxidase subunit II (COX2) & 2758 \\
\hline mtAll-F41 & Sequencing & CTGCGGAGCAAACCATAGC & tRNA-Lys & \\
\hline mtAll-F42 & Sequencing & AAGAAGTCATGTGTTTTCCCTTG & ATP synthase F0 subunit 8 (ATP8) & \\
\hline mtAll-F43 & Sequencing & AAATCTCACTAGCCCATCTTCTCC & ATP synthase F0 subunit 6 (ATP6) & \\
\hline mtAll-F45 & Sequencing & TGGGCTGTCCCATCCCTAGGT & Cytochrome c oxidase subunit III (COX3) & \\
\hline mtAll-R4 & PCR, Sequencing & TGAGTCGAAATCAYTTGTT & tRNA-Gly & \\
\hline mtAll-R41 & Sequencing & TAAAGAAATAGGATCCTCATCAGTAAA & NADH dehydrogenase subunit 3 (ND3) & \\
\hline mtAll-R42 & Sequencing & TGGTGAGCTCAGGTAATAGTCACTC & tRNA-Arg & \\
\hline mtAll-R43 & Sequencing & AGATAATGCTCCGGTAAGAGGTC & & \\
\hline Set 5 & & & & \\
\hline mtAll-F5 & PCR, Sequencing & GCCATCCAAGCTAATAATACAAGTCTA & NADH dehydrogenase subunit $3(N D 3)$ & 2145 \\
\hline mtAll-F51 & Sequencing & CTCCAATACCTACGGACTAGACTACG & tRNA-Arg & \\
\hline mtAll-F52 & Sequencing & ACATTATATTTGAAACAACGCTTATC & NADH dehydrogenase subunit $4 \mathrm{~L}$ (ND4L) & \\
\hline mtAll-F53 & Sequencing & AATACCTCTATATGGTCTCCACCTATG & NADH dehydrogenase subunit 4 (ND4) & \\
\hline mtAll-F54 & Sequencing & TTTTAGCACGAGGCTTACAAAC & tRNA-His & \\
\hline mtAll-R5 & PCR, Sequencing & AAGCAATTTGTCTTGTTGTTTTGT & tRNA-Ser & \\
\hline mtAll-R51a & Sequencing & CGGGTAAATGAAGGTTTAATGG & tRNA-Leu & \\
\hline mtAll-R52 & Sequencing & TGGTAATTCGTAGTATCCCATACCC & NADH dehydrogenase subunit 5 (ND5) & \\
\hline mtAll-R53 & Sequencing & AGGAAGTATGACCCTGCGTTTA & & \\
\hline mtAll-R54 & Sequencing & TGTTAGAGGTAAATCAGGCAAGG & & \\
\hline Set 6 & & & & \\
\hline ND56-F1d & PCR, Sequencing & TGGTGCAACTCCARATAAAAGTAA & NADH dehydrogenase subunit 5 (ND5) & 2149 \\
\hline ND56-3F2 & Sequencing & AACAGAYGCTAACACAGCAGCTCT & NADH dehydrogenase subunit 6 (ND6) & \\
\hline ND56-4F2 & Sequencing & CCTAYATCAACACCTGAGCACTA & tRNA-Glu & \\
\hline ND56-45F2 & Sequencing & ATTTACRGTAGCAACAGAACTTAACAA & Cytochrome b (CYTB) & \\
\hline ND56-5F2 & Sequencing & CCCCAGAATAATCCTCACG & & \\
\hline ND56-R1A & PCR, Sequencing & TYACATCTCGGCAAATATGG & & \\
\hline ND56-5R2 & Sequencing & CTTGAGGGTCAAATGTTGTA & & \\
\hline ND56-35R2 & Sequencing & GCACAGATAGCTGTRAATAAAGTAGTAAT & & \\
\hline ND56-45R2 & Sequencing & TGTTAAGTTCTGTTGCTACYGTAAATC & & \\
\hline Set 7 & & & & \\
\hline CBCR-F1d & PCR, Sequencing & ACCATGACTAATGATCTGAAAAACC & tRNA-Glu & 1938 \\
\hline CBCR-F2 & Sequencing & TTCATAGGATAYGTCCTTCC & Cytochrome b (CYTB) & \\
\hline CBCR-F3 & Sequencing & KTACGCCATYCTACGATC & tRNA-Thr & \\
\hline CBCR-F5 & Sequencing & ATTACAATGGTCTTGTAAGCCATAAA & tRNA-Pro & \\
\hline CBCR-F6 & Sequencing & GATAAACCATAGTCTTACATAGCACAT & D-Loop & \\
\hline CBCR-R1d & PCR, Sequencing & CTCAGACGGCCATAGCTGA & & \\
\hline CBCR-R3 & Sequencing & GGACRCCTCCTAGTTTGTTTGGTA & & \\
\hline CBCR-R4 & Sequencing & CTTCCCTGAATACCCTTAGAAA & & \\
\hline CBCR-R5 & Sequencing & GCTTTAATGTGCTATGTAAGACTATGG & & \\
\hline CBCR-R52 & Sequencing & AAGCCTCCTCARATTCATTCTACTA & & \\
\hline Set 8 & & & & \\
\hline mtAll-F8 & PCR, Sequencing & ACCCAAARCTGANATTCT & tRNA-Pro & 1731 \\
\hline mtAll-F82 & Sequencing & GTTAATGTAGCTTAAAACAAAAGCAA & D-Loop & \\
\hline mtAll-R8 & PCR, Sequencing & TGTTACGACTTGTCTCCTCT & tRNA-Phe & \\
\hline mtAll-R81 & Sequencing & GACTGAATTAGCAAG & $12 \mathrm{~S}$ ribosomal RNA & \\
\hline mtAll-R82 & Sequencing & ACGCCGTATGCTTATTAATTTGG & & \\
\hline
\end{tabular}

PCR amplicon sizes are based on NC_000934 


\section{Supporting Information Figure S1. Amino acid alignments for mitochondrial proteins}

across elephantids. The translated codons of 13 protein coding genes in elephantid mitogenomes were aligned for two forest elephants (DS1534 and SL0001) and seven other elephantids (GenBank numbers: NC_000934, AB443879, DQ316069, AJ428946, NC_005129, EU153453, and NC_007596) using Geneious v5.4 (Drummond et al. 2011). Conserved amino acids are shown as dots below a consensus sequence. Colors indicate the hydrophobicity of amino acid residues, with red being highly hydrophobic and blue highly hydrophilic. With few exceptions the differences between the forest elephants and the other elephantids fell into the following categories: (1) amino acid differences that did not involve change from a highly hydrophobic to a highly hydrophilic residue sequence, or vice versa; (2) amino acid substitutions that were also present in at least one other elephantid species; or (3) alternative stop codon present in at least one other elephantid species. The following comprise the only exceptions: For forest elephant DS1534, ATP6 appears to rely on an alternative start codon from the other elephantids, ND4L has a different stop codon from the other elephantids, and ND4 has Gln instead of Leu in the second amino acid position. For forest elephant SL0001, ND3 relies on an alternative start codon from the other elephantids. Both forest elephants have Tyr instead of His at position 312 of CYTB. Abbreviations: LCY: Loxodonta cyclotis, African forest elephant; LAF: Loxodonta africana, African savanna elephant; EMA: Elephas maximus, Asian elephant; MPR: Mammuthus primigenius, woolly mammoth.

Reference: Drummond AJ, Ashton B, Buxton S, Cheung M, Cooper A, Duran C, Field M, Heled J, Kearse M, Markowitz S, Moir R, Stones-Havas S, Sturrock S, Thierer T, Wilson A (2011) Geneious v5.4, Available from http://www.geneious.com/ 


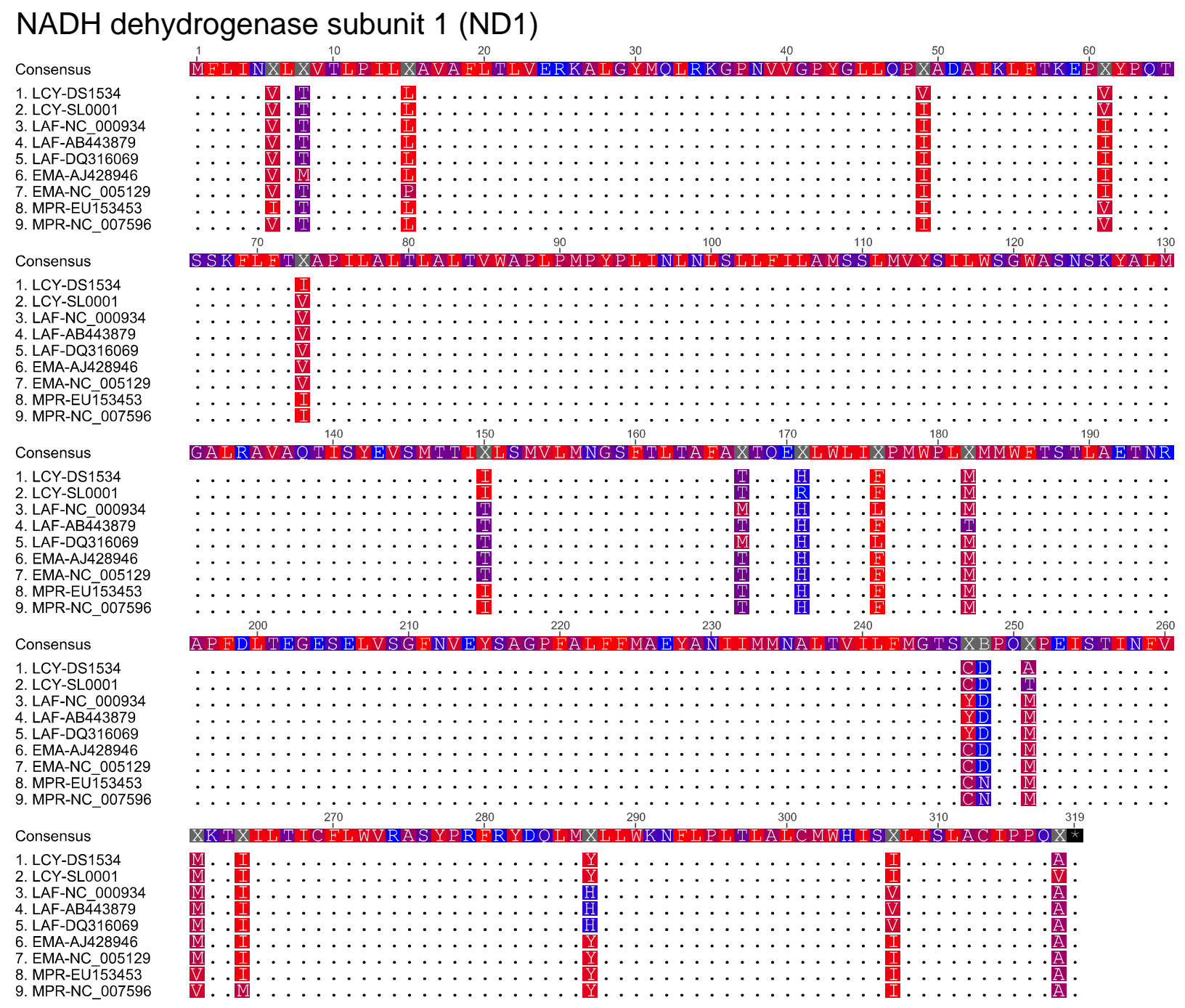




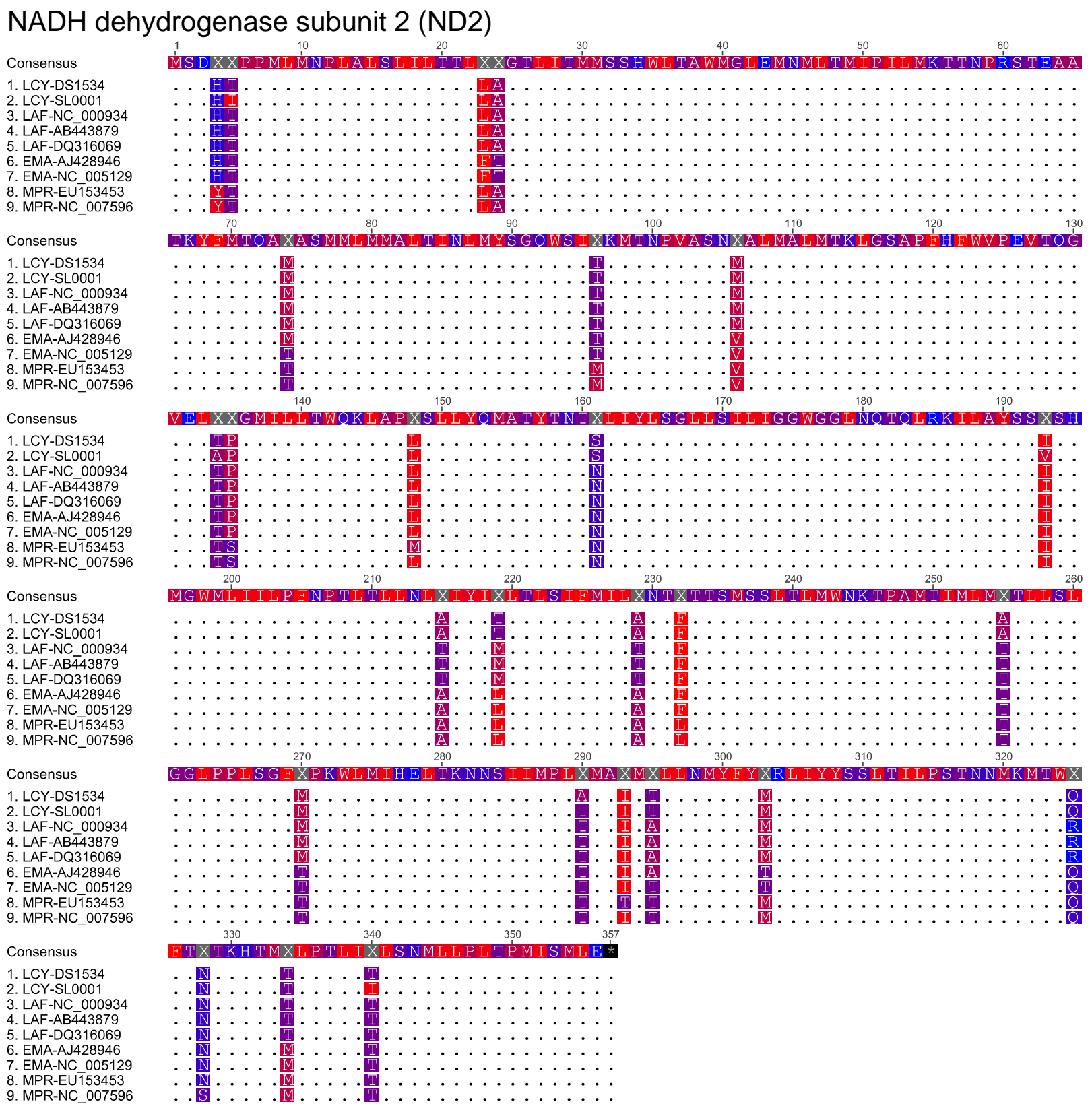


Cytochrome c oxidase subunit I (COX1) Consensus
1. LCY-DS1534
2. LCY-SL0001
3. LAF-NC_000934
4. LAF-AB443879
5. LAF-DQ316069
6. EMA-AJ428946
7. EMA-NC_005129
8. MPR-NC_007596
9. MPR-EU153453

Consensus

1. LCY-DS1534

2. LCY-SL0001

4. LAF-AB 443879

5. LAF-DQ316069

6. EMA-AJ428946

7. EMA-NC_005129

9. MPR-EU 153453

Consensus

1. LCY-DS1534

2. LCY-SL0001

3. LAF-NC_000934

4. LAF-AB4443879

5. LAF-DQ316069

6. EMA-AJ428946

7. EMA-NC_005129

9. MPR-EU153453

Consensus

1. LCY-DS1534

2. LCY-SL0001

3. LAF-NC_000934

4. LAF-AB 443879

5. LAF-AB443879

5. EMF-DQ316069

7. EMA-NC_005129

8. MPR-NC 007596
9. MPR-EU153453

Consensus

1. LCY-DS1534

2. LCY-SL0001

3. LAF-NC_000934

5. LAF-AB443879

5. LAF-DQ316069

6. EMA-AJ428946
7. EMA-NC 005129

7. EMA-NC_005129

9. MPR-EU153453

Consensus

1. LCY-DS1534

2. LCY-SL0001

3. LAF-NC_000934

4. LAF-AB $4 \overline{4} 3879$

5. LAF-DQ316069

6. EMA-AJ428946

7. EMA-NC_005129

8. MPR-NC_007596

9. MPR-EU153453

Consensus

1. LCY-DS1534

2. LCY-SL0001

3. LAF-NC_000934

4. LAF-AB 443879

5. LAF-DQ316069

6. EMA-AJ428946

7. EMA-NC_005129

9. MPR-EU153453

Consensus

1. LCY-DS1534

2. LCY-SL0001

3. LAF-NC_000934

4. LAF-AB 443879

5. LAF-DQ316069

6. EMA-AJ428946

7. EMA-NC_005129

8. MPR-NC_007596

9. MPR-EU153453

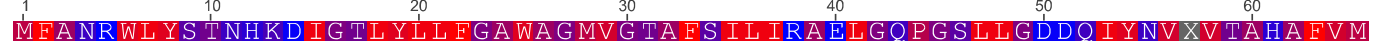

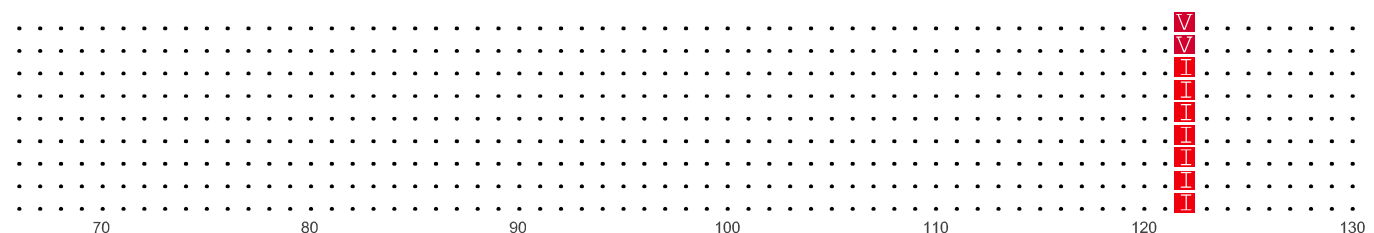

IF FMVMP IMIGGFG 'NWL IPLMIGA

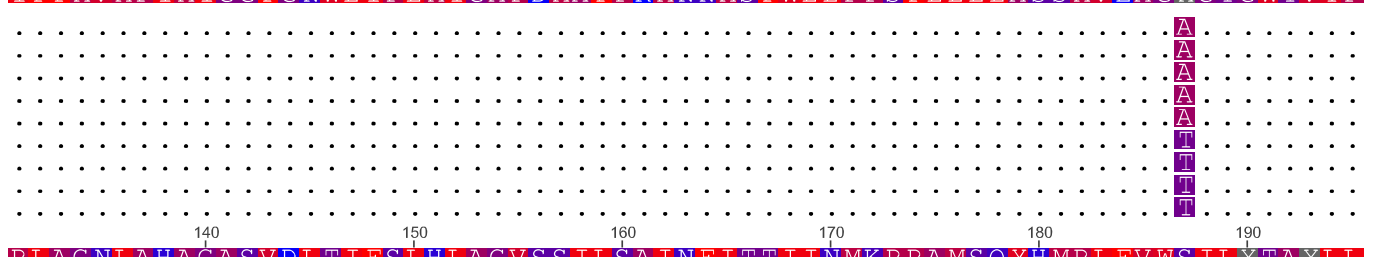

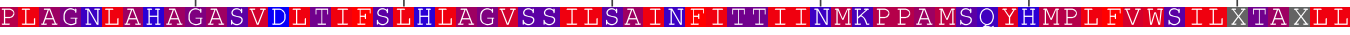

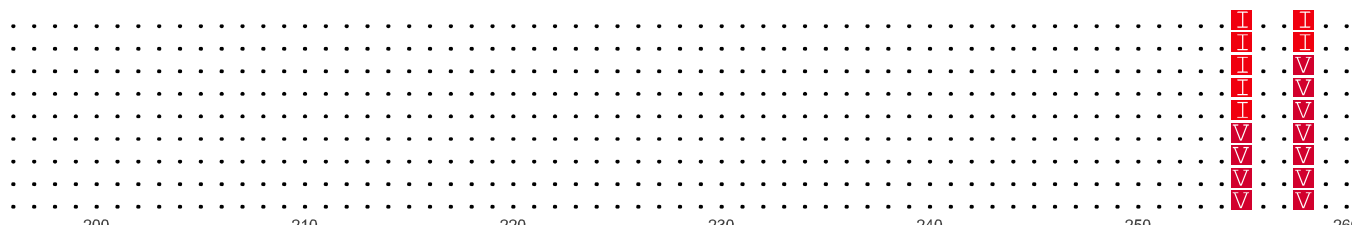

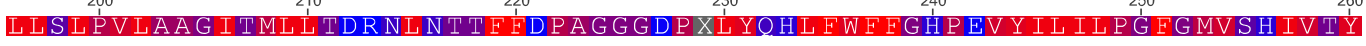

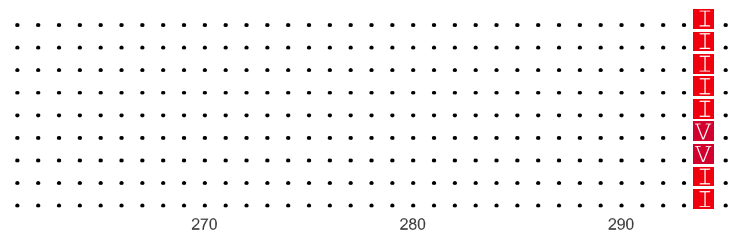

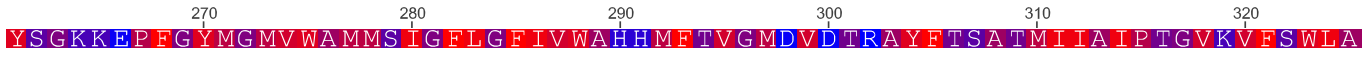

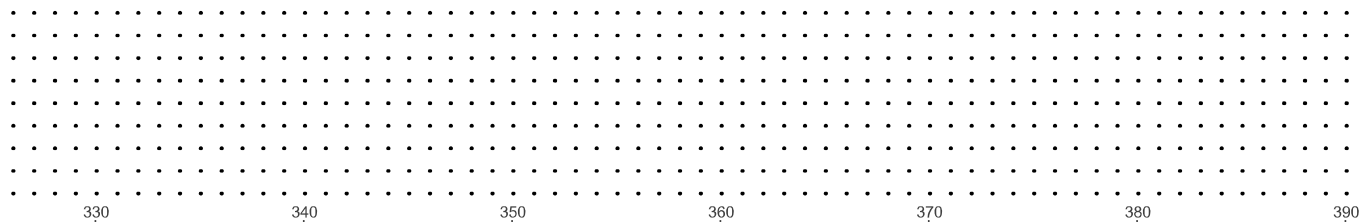

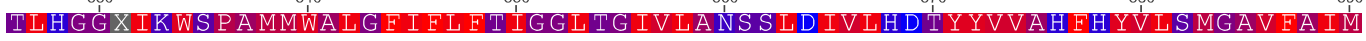
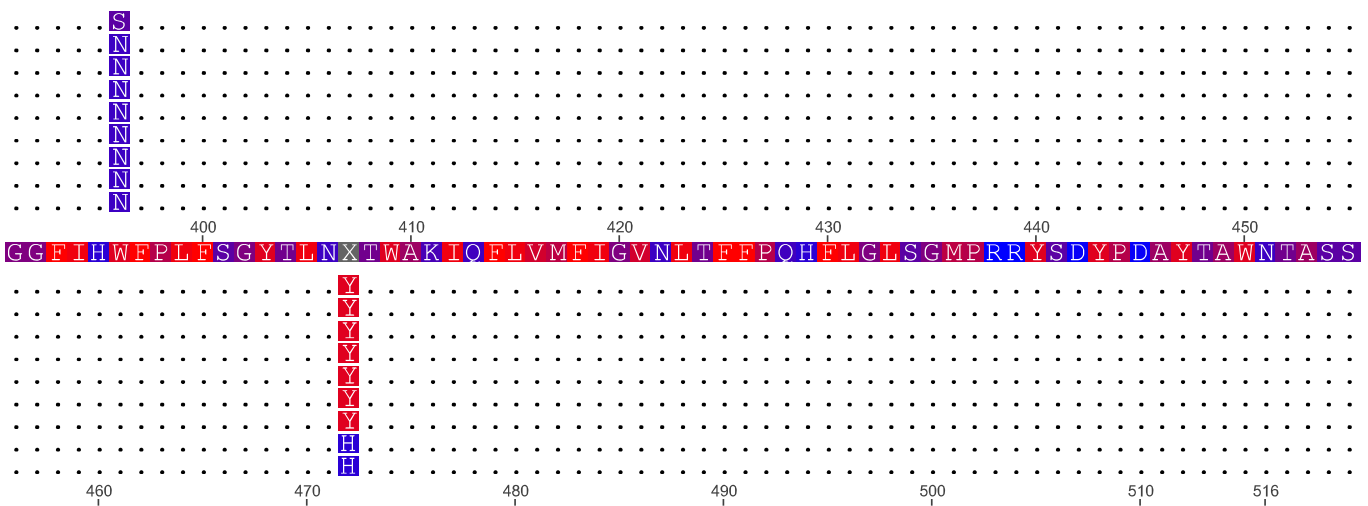

MGSF ISLVAV L LV '

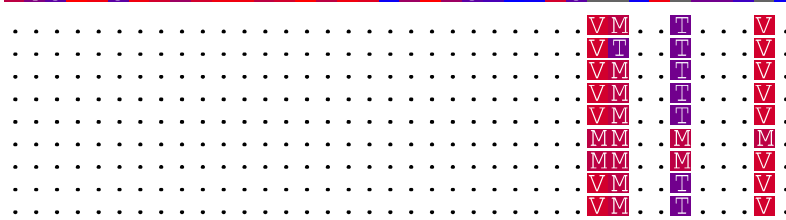




\section{Cytochrome c oxidase subunit II (COX2)}

Consensus
1. LCY-DS1534
2. LCY-SL0001
3. LAF-NC_000934
4. LAF-AB443879
5. LAF-DQ316069
6. EMA-AJ428946
7. EMA-NC_005129
8. MPR-EU153453
9. MPR-NC_007596
Consensus
1. LCY-DS1534
2. LCY-SL0001
3. LAF-NC_000934
4. LAF-AB443879
5. LAF-DQ316069
6. EMA-AJ428946
7. EMA-NC_005129
8. MPR-EU153453
9. MPR-NC_007596

Consensus

1. LCY-DS1534

2. LCY-SL0001

4. LAF-NC 000934

5. LAF-AB443879

5. LAF-DQ316069

7. EMA-AJ428946

7. EMA-NC_005129

8. MPR-EU153453
9. MPR-NC_007596

Consensus

1. LCY-DS1534

2. LCY-SL0001

3. LAF-NC_000934

4. LAF-AB 443879

5. LAF-DQ316069
6. EMA-AJ428946

6. EMA-AJ428946
7. EMA-NC 005129

8. MPR-EU153453

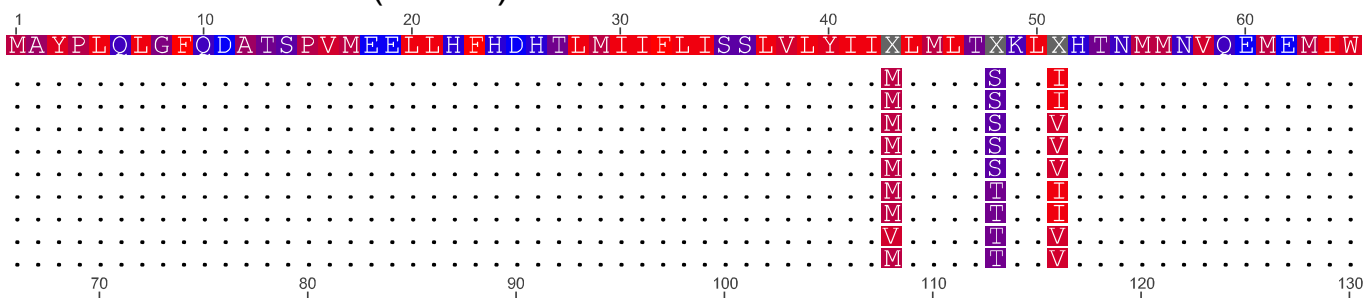

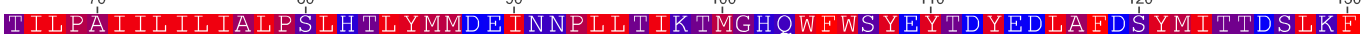

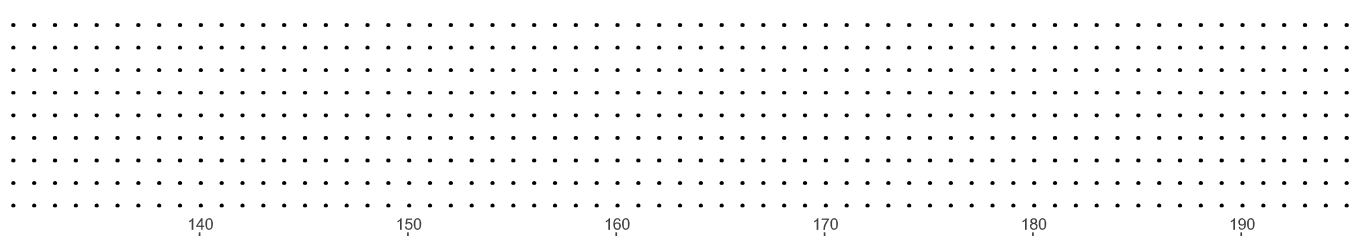

GELRLLEVD NRMVLPTDLPXRVLVSSEDVLHS WAVPSLGLKTDA IPGRLNOVTLTSMRPGLFYGO

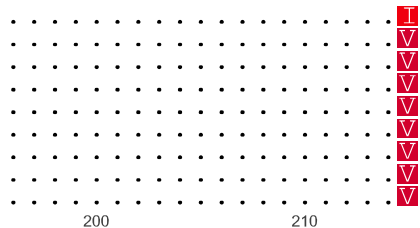

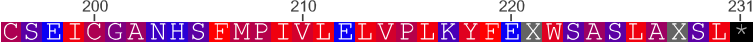

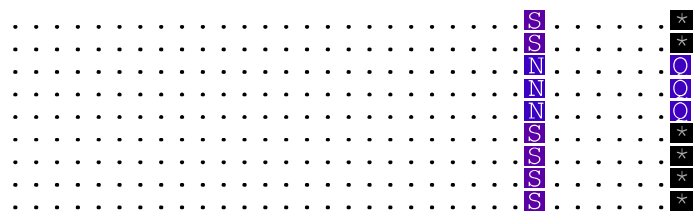




\section{ATP synthase F0 subunit 8 (ATP8)}

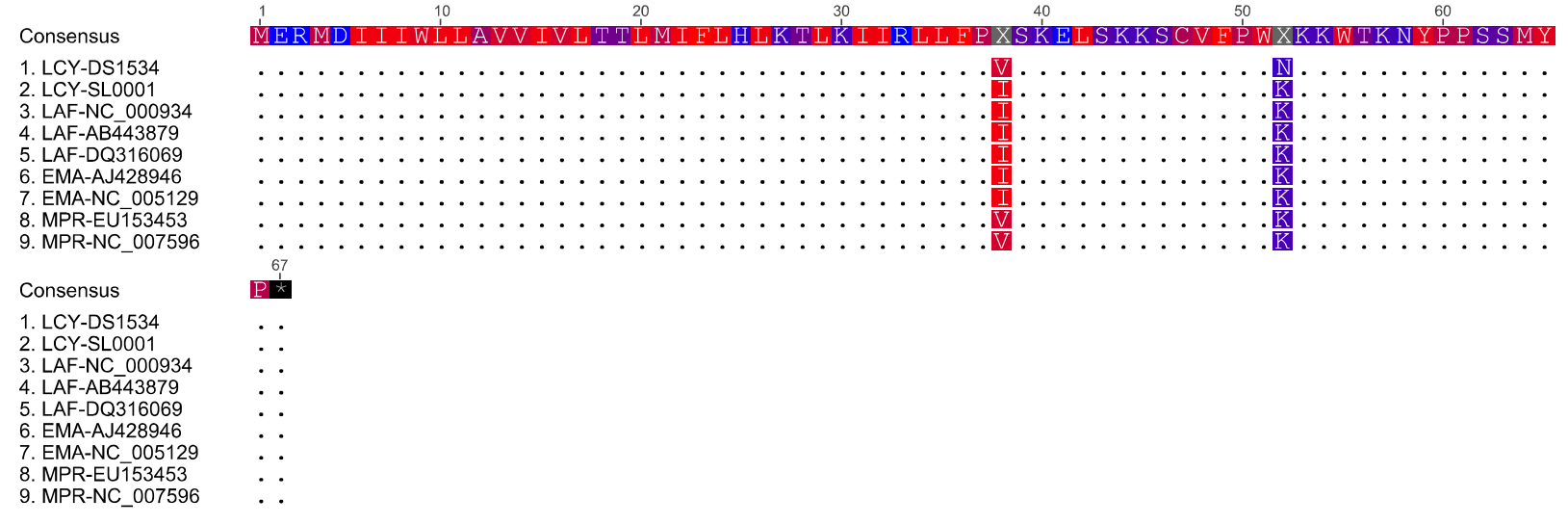


ATP synthase F0 subunit 6 (ATP6)

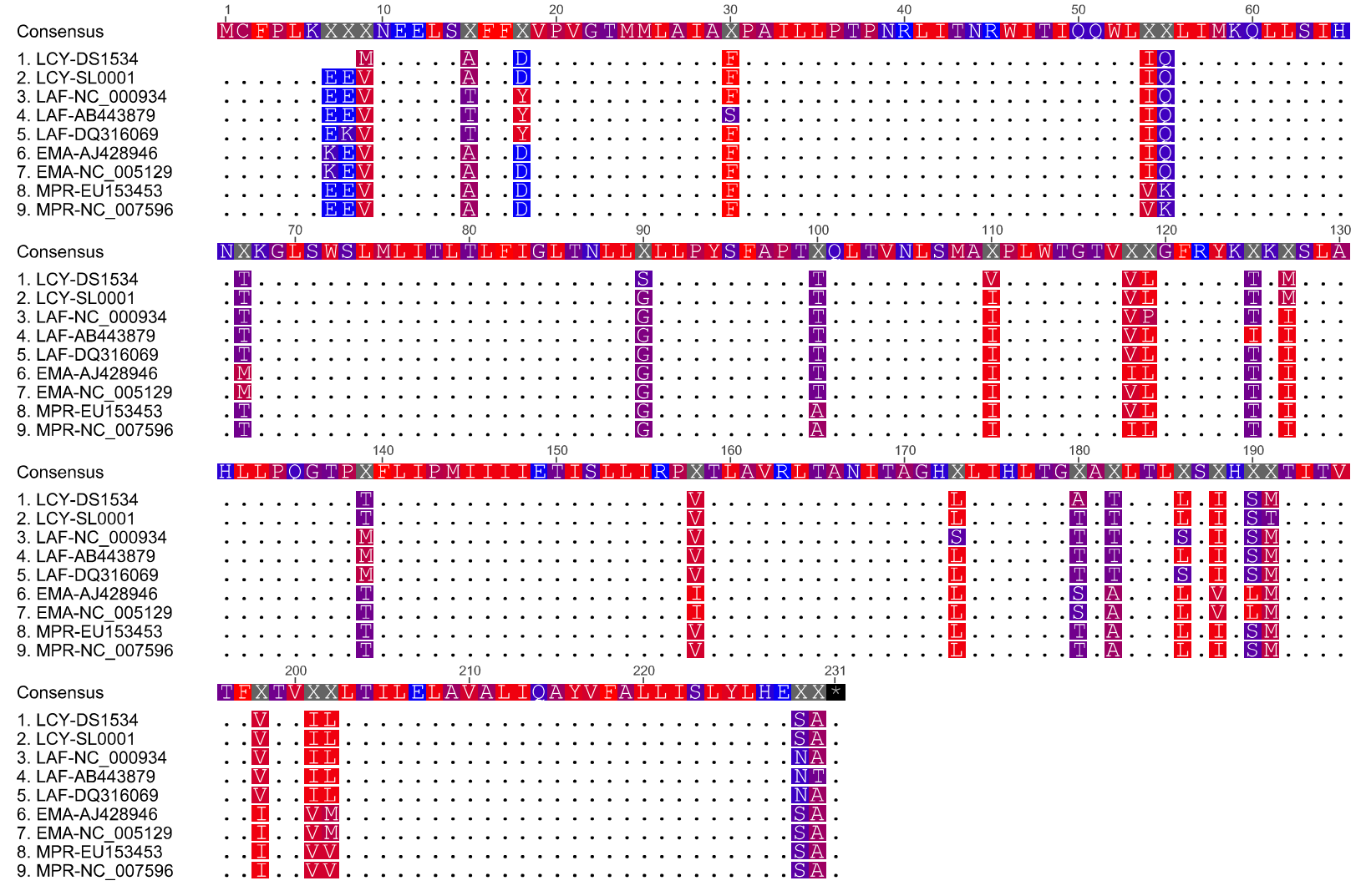




\section{Cytochrome c oxidase subunit III (COX3)}

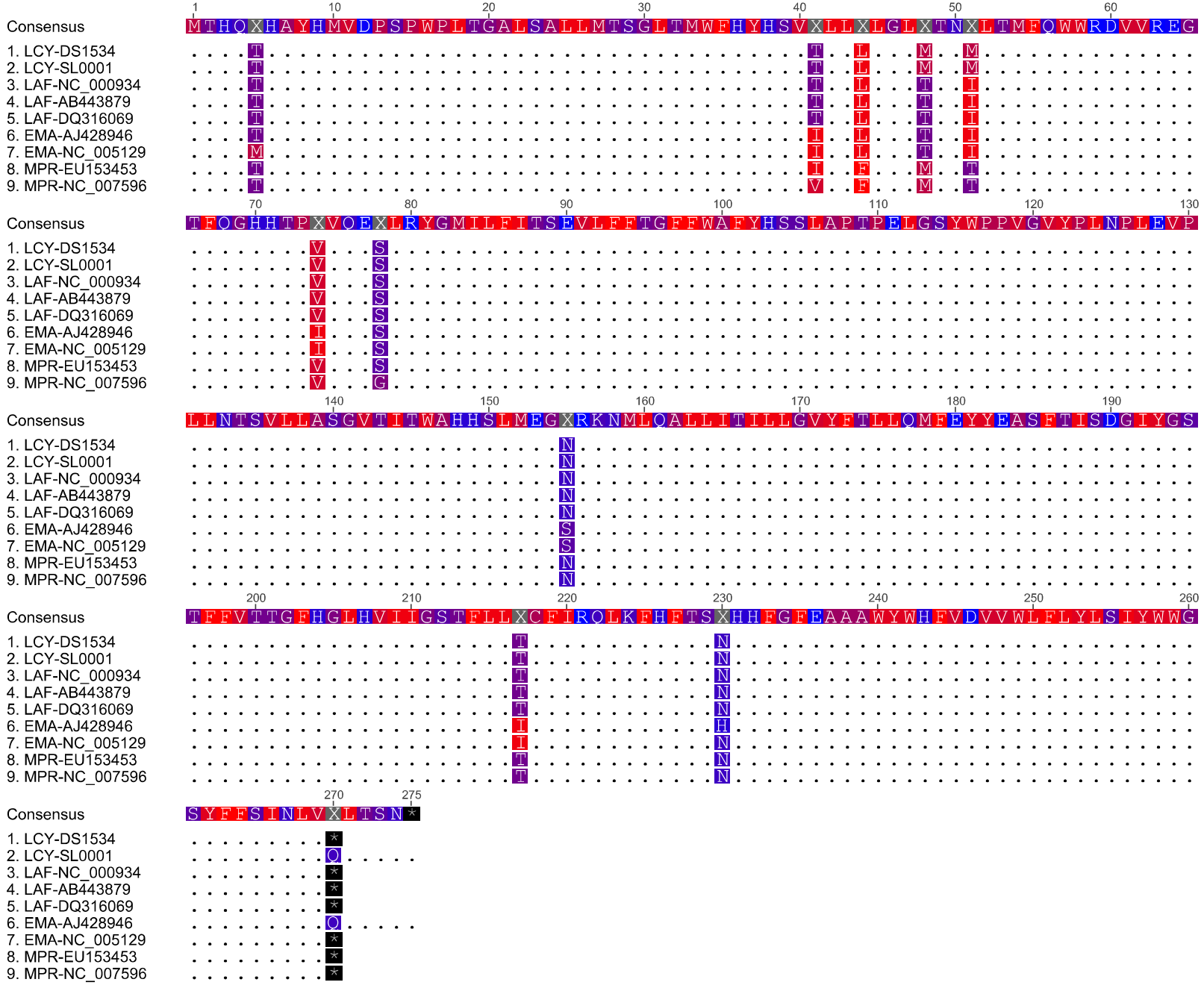


NADH dehydrogenase subunit 3 (ND3)

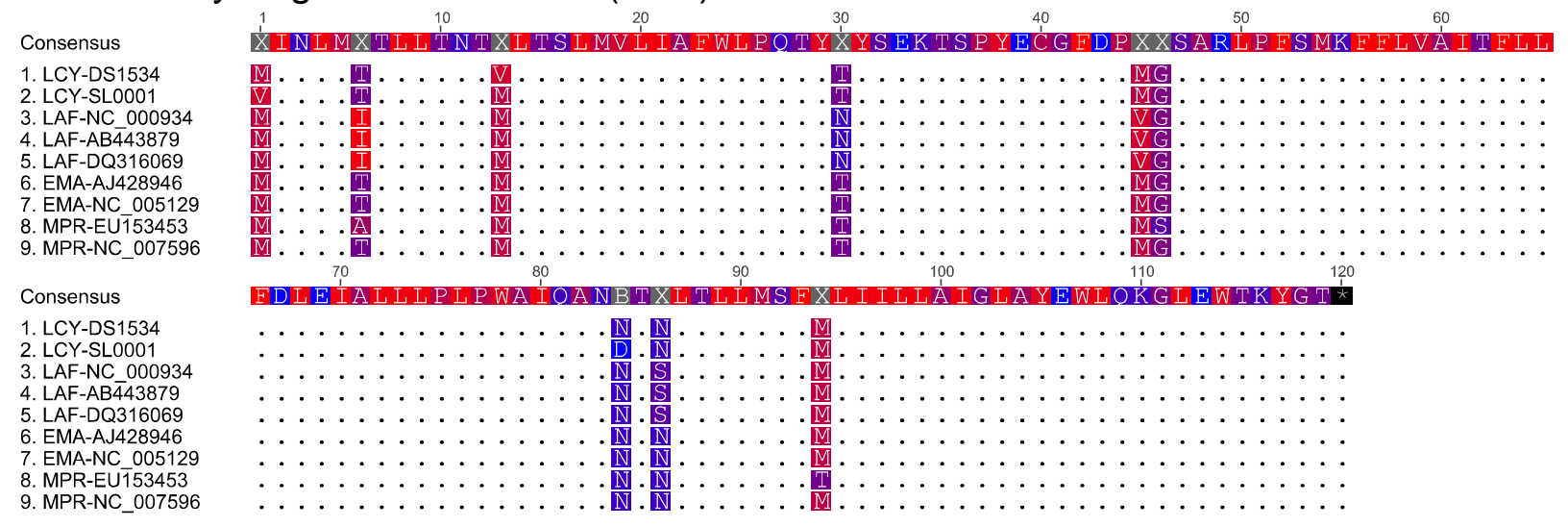


NADH dehydrogenase subunit 4L (ND4L)

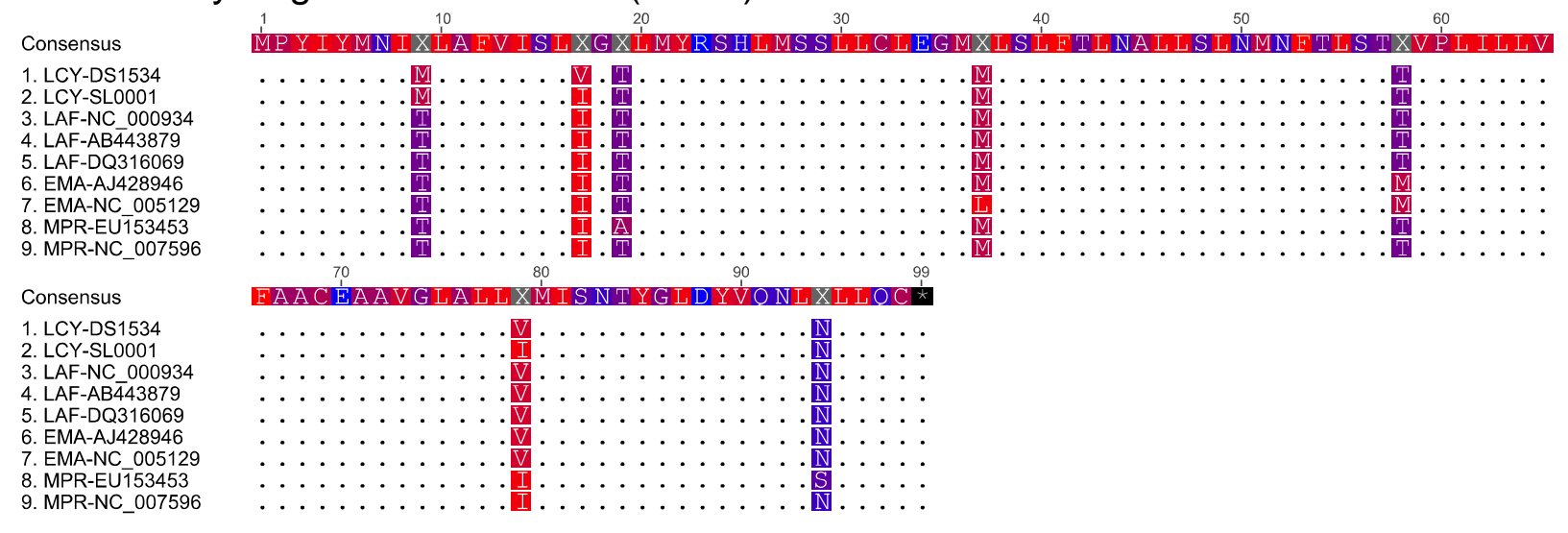


NADH dehydrogenase subunit 4 (ND4)

Consensus

1. LCY-DS1534

2. LCY-SL0001

3. LAF-NC 000934

4. LAF-AB $\overline{4} 43879$

5. LAF-DQ316069

6. EMA-AJ428946

7. EMA-NC 005129

8. MPR-EU153453

9. MPR-NC 007596

Consensus

1. LCY-DS1534

2. LCY-SL0001

3. LAF-NC_000934

4. LAF-AB $\overline{4} 43879$

5. LAF-DQ316069

6. EMA-AJ428946

7. EMA-NC 005129

8. MPR-EU153453

9. MPR-NC 007596

Consensus

1. LCY-DS1534

2. LCY-SL0001

3. LAF-NC 000934

4. LAF-AB 443879

5. LAF-DQ316069

6. EMA-AJ428946

7. EMA-NC 005129

8. MPR-EU 153453

9. MPR-NC_007596

Consensus

1. LCY-DS1534

3. LAF-NC 000934

4. LAF-AB 443879

5. LAF-DQ316069

6. EMA-AJ428946

7. EMA-NC 005129

8. MPR-EU153453

9. MPR-NC 007596

Consensus

1. LCY-DS1534

2. LCY-SL0001

3. LAF-NC 000934

4. LAF-AB $\overline{4} 43879$

5. LAF-DQ316069

6. EMA-AJ428946

7. EMA-NC 005129

8. MPR-EU 153453

9. MPR-NC 007596

Consensus

1. LCY-DS1534

2. LCY-SL0001

3. LAF-NC 000934

4. LAF-AB 443879

5. LAF-DQ316069

6. EMA-AJ428946

7. EMA-NC 005129

8. MPR-EU 153453

9. MPR-NC_007596

Consensus

1. LCY-DS1534

2. LCY-SL0001

3. LAF-NC 000934

4. LAF-AB 443879

5. LAF-DQ316069

6. EMA-AJ428946

7. EMA-NC 005129

8. MPR-EU1153453

9. MPR-NC_007596

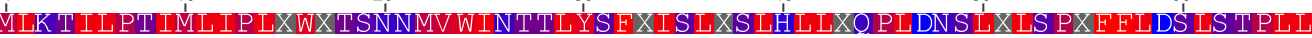
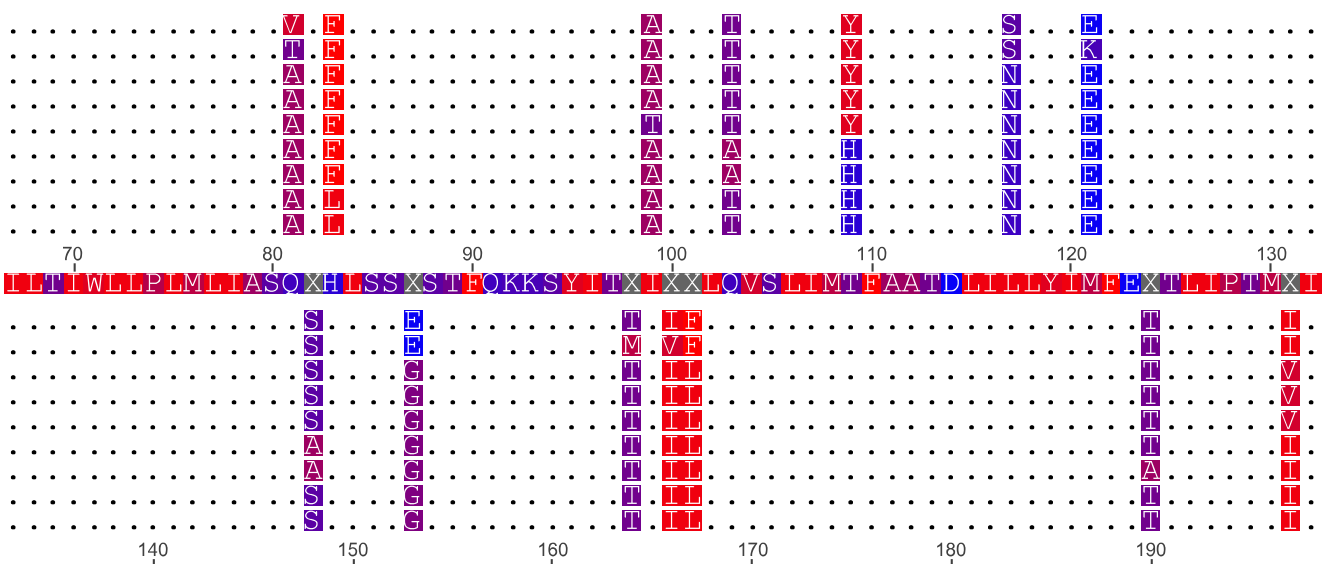

IIRWGNOLER LBAGS YF LFYTLXGS LPLLVTLMLION'LGS LNLMIMLPY LXKXIBNLW'INMILWLT

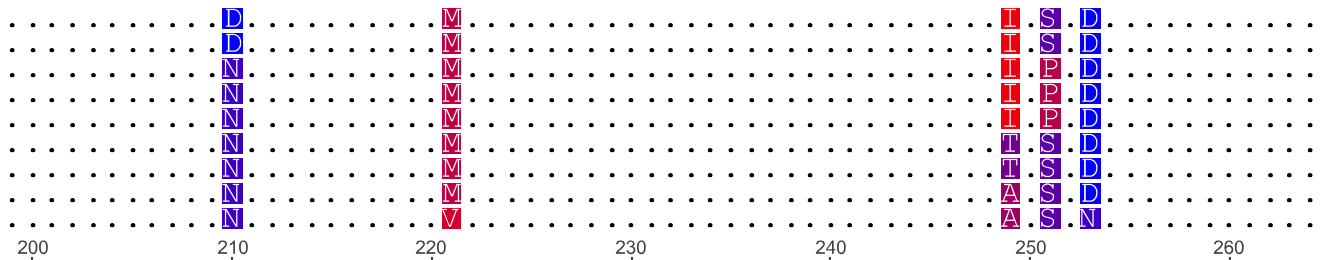

CXMA FMVK MP L YG LHLW LPK AHVEAP I AGS MV LAAI L LK LGGYGMLRITI L LDPLTXHMYYP F LML

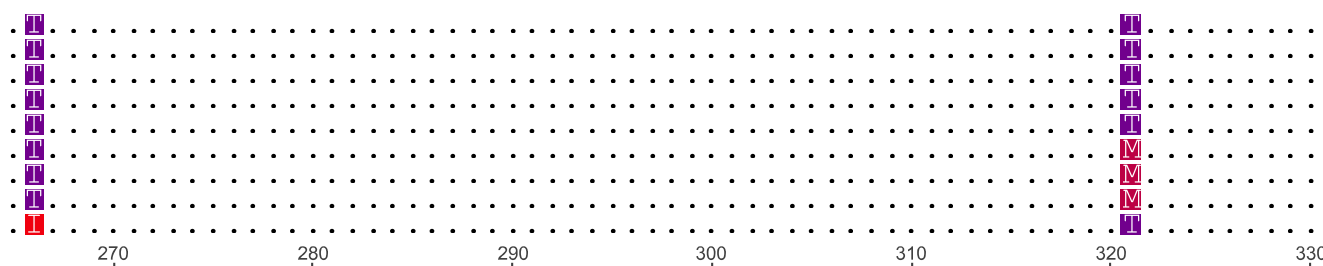

S LWG MV MXSS I CLRO'TDMKS LIAYS S'VSHMALVI IA IMLOTPWS FMGA LX LMIAHGLTSS MLFCLA

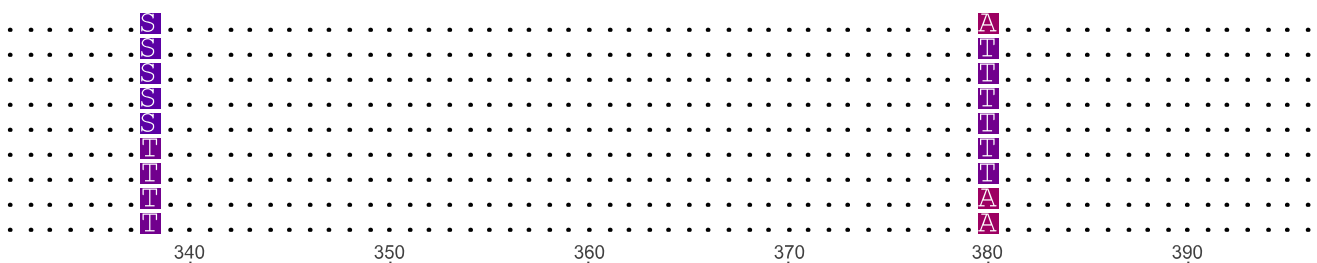

NSNYER IHXR'TMI LARG LQT'LLP LMAXWW'LAS LXNMAPPPTINLXGELLI ITTS FSWSN XTI FPM
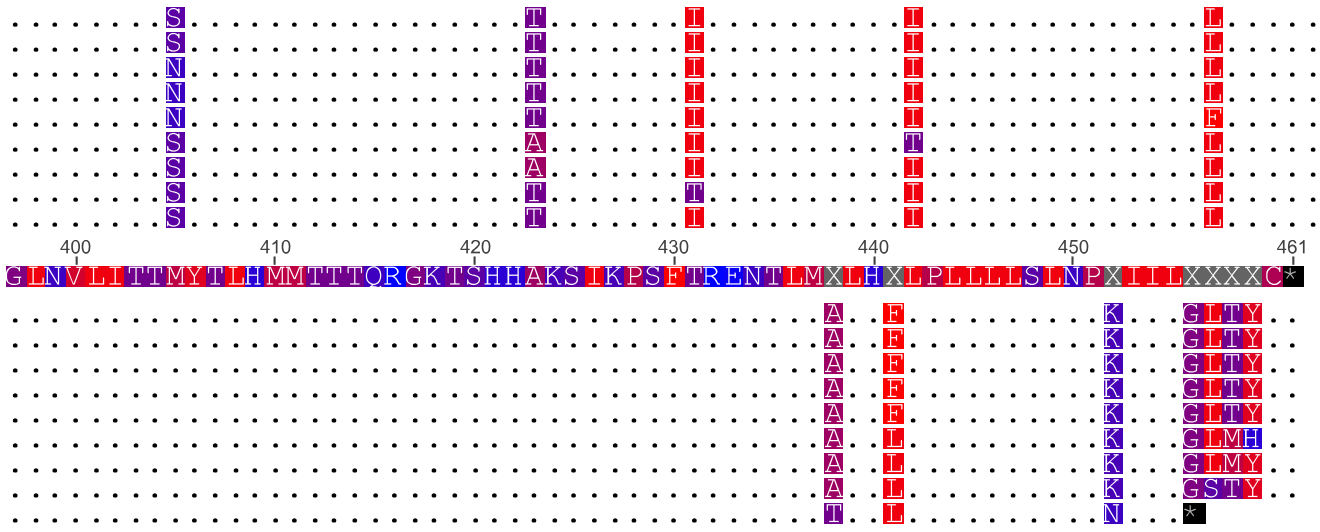
NADH dehydrogenase subunit 5 (ND5)

Consensus
1. LCY-DS1534
2. LCY-SL0001
3. LAF-NC_000934
4. LAF-AB443879
5. LAF-DQ316069
6. EMA-AJ428946
7. EMA-NC_005129
8. MPR-EU153453
9. MPR-NC_007596

Consensus

1. LCY-DS1534

2. LCY-SL0001
3. LAF-NC 000934

4. LAF-AB 443879

5. LAF-DQ316069

6. EMA-AJ428946

8. MPR-EU153453

9. MPR-NC_007596

Consensus

1. LCY-DS1534

2. LCY-SL0001

3. LAF-NC_000934

4. LAF-AB 443879

5. LAF-DQ316069

6. EMA-AJ428946

7. EMA-NC_005129

9. MPR-NC_007596

Consensus

1. LCY-DS1534

2. LCY-SL0001

3. LAF-NC_000934

4. LAF-AB443879

5. LAF-DQ316069

6. EMA-AJ428946

7. EMA-NC 005129

9. MPR-NC_007596

Consensus

1. LCY-DS1534

2. LCY-SL0001

2. LCY-SL0001

4. LAF-NC_000934

4. LAF-AB443879

5. LAF-DQ316069

6. EMA-AJ428946

7. EMA-NC 005129

9. MPR-NC_007596

Consensus

1. LCY-DS1534

2. LCY-SL0001

3. LAF-NC 000934

5. LAF-AB443879

6. EMA-AJ428946

7. EMA-NC_005129

8. MPR-EU153453

9. MPR-NC_007596

Consensus

1. LCY-DS1534

2. LCY-SL0001

3. LAF-NC_000934

4. LAF-AB 443879

5. LAF-DQ316069

6. EMA-AJ428946

7. EMA-NC 005129

9. MPR-NC_007596

Consensus

1. LCY-DS1534

2. LCY-SL0001

3. LAF-NC_000934

4. LAF-AB443879

5. LAF-DQ316069

6. EMA-AJ428946

7. EMA-NC_005129

8. MPR-EU153453

9. MPR-NC_007596

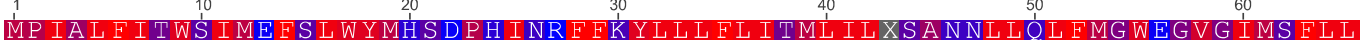
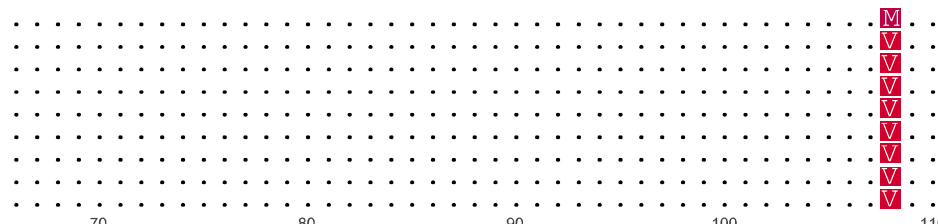

IS WWHGR TDANTAA 'ंOAML YNR I GDMG F IMMMA W'
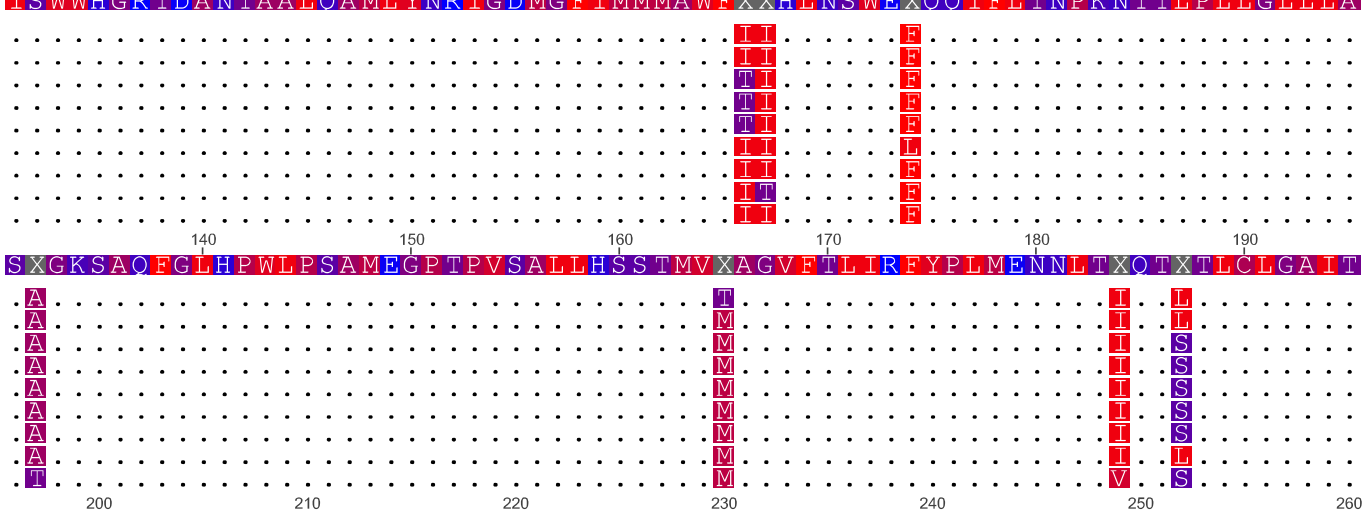

TLFTÁ ICALTONDIKKI IALSTSSOLGLMMVTIG INOPXLA FXHMC THAF FKAMLFLSSGSI IH
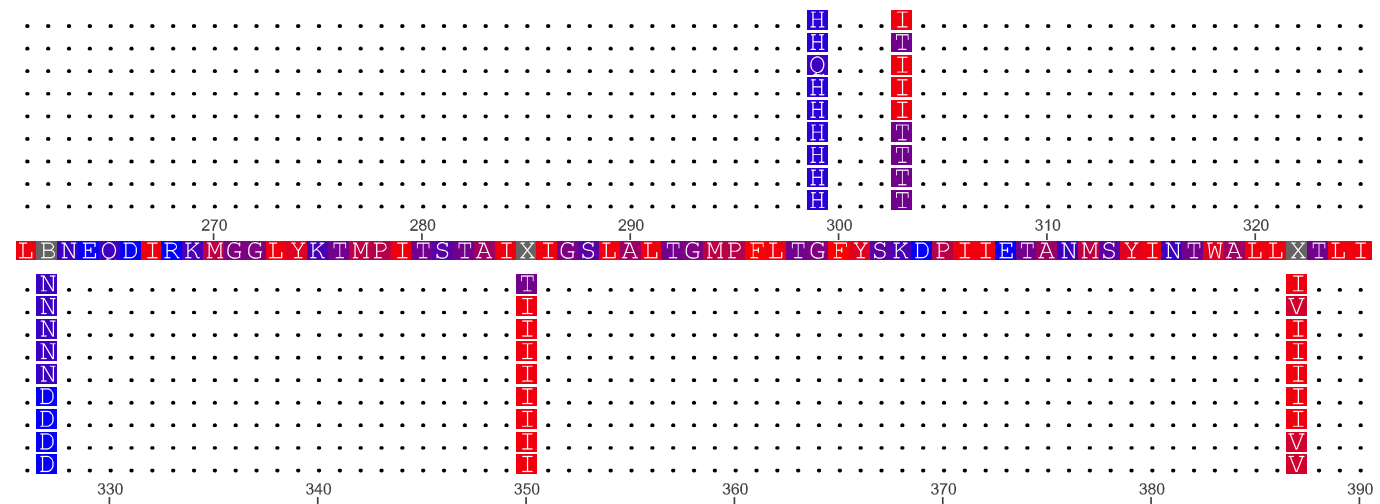

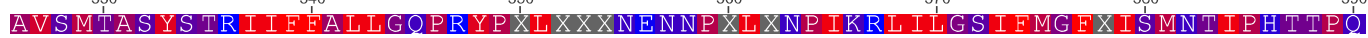

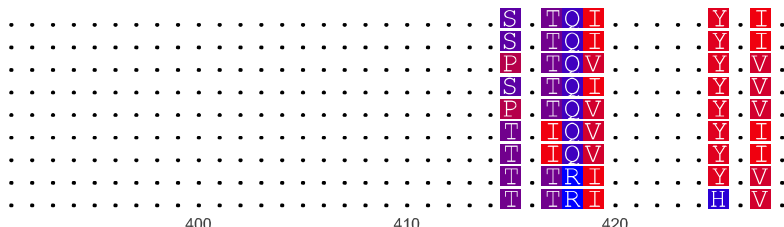

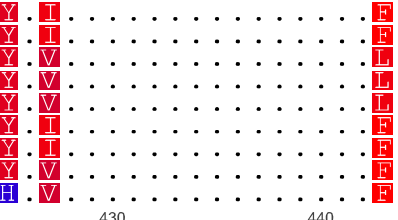

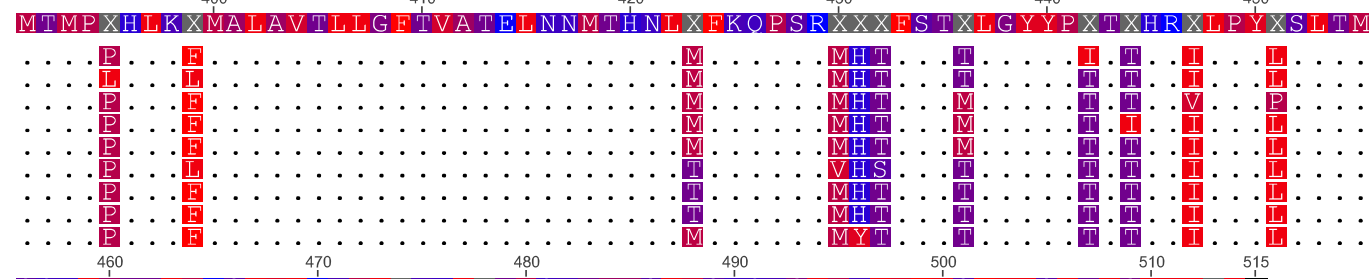

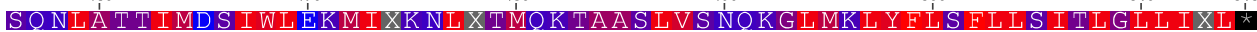

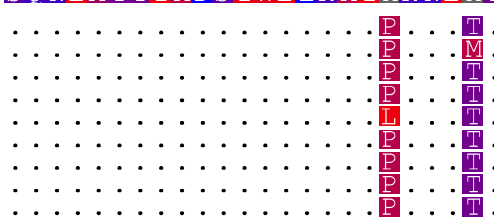




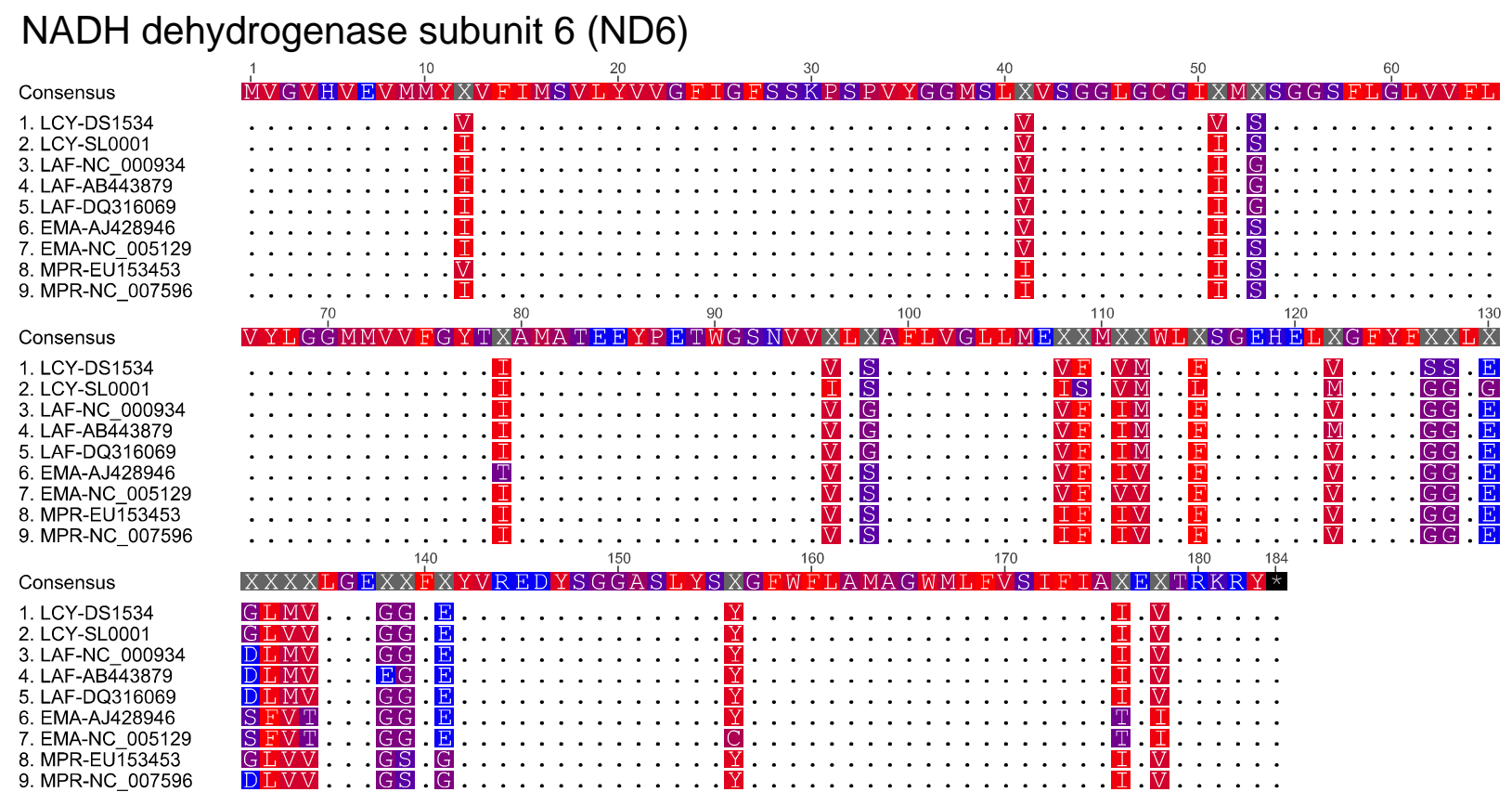




\section{Cytochrome b (CYTB)}

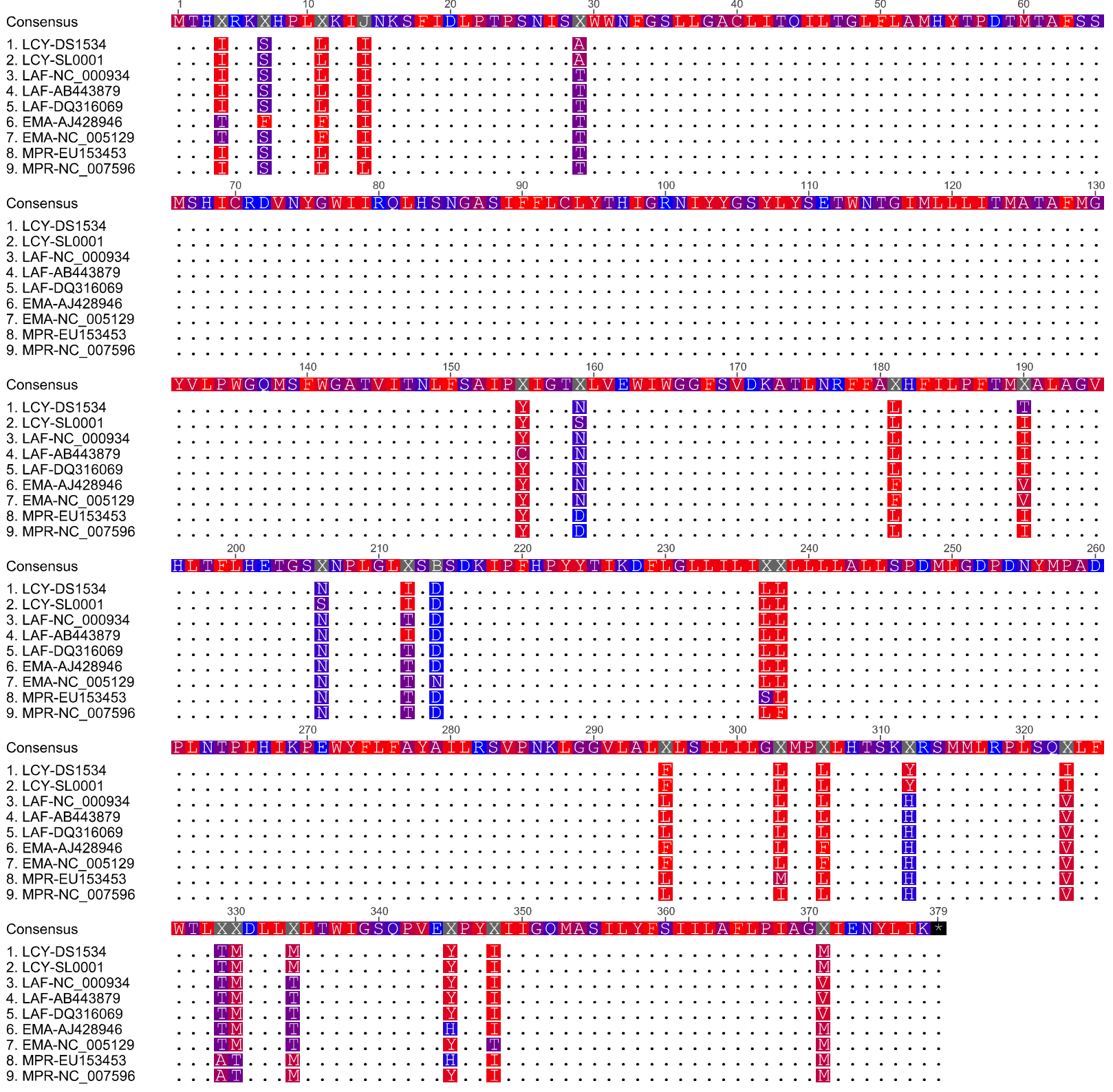

